ESAIM: M2AN 47 (2013) 1797-1820

DOI: $10.1051 / \mathrm{m} 2 \mathrm{an} / 2013089$
ESAIM: Mathematical Modelling and Numerical Analysis

www.esaim-m2an.org

\title{
AUTOMATIC SIMPLIFICATION OF DARCY'S EQUATIONS WITH PRESSURE DEPENDENT PERMEABILITY
}

\author{
Etienne Ahusborde ${ }^{1}$, Mejdi Azä̈ez ${ }^{2}$, Faker Ben Belgacem ${ }^{3}$ \\ And Christine Bernardi ${ }^{4}$
}

\begin{abstract}
We consider the flow of a viscous incompressible fluid in a rigid homogeneous porous medium provided with mixed boundary conditions. Since the boundary pressure can present high variations, the permeability of the medium also depends on the pressure, so that the model is nonlinear. A posteriori estimates allow us to omit this dependence where the pressure does not vary too much. We perform the numerical analysis of a spectral element discretization of the simplified model. Finally we propose a strategy which leads to an automatic identification of the part of the domain where the simplified model can be used without increasing significantly the error.
\end{abstract}

Mathematics Subject Classification. 76S05, 65N35.

Received October 19, 2011.

Published online October 7, 2013.

\section{INTRODUCTION}

Let $\Omega$ be a bounded connected domain in $\mathbb{R}^{d}, d=2$ or 3 , with a Lipschitz-continuous boundary $\partial \Omega$. We assume that this boundary is divided into two disjoint parts $\Gamma_{(p)}$ and $\Gamma_{(f)}$ such that $\partial \Gamma_{(p)}$ and $\partial \Gamma_{(f)}$ are Lipschitz-continuous submanifolds of $\partial \Omega$. We are interested in studying the following model, suggested by Rajagopal [24],

$$
\begin{cases}\alpha(p) \boldsymbol{u}+\operatorname{grad} p=\boldsymbol{f} & \text { in } \Omega, \\ \operatorname{div} \boldsymbol{u}=0 & \text { in } \Omega, \\ p=p_{0} & \text { on } \Gamma_{(p)}, \\ \boldsymbol{u} \cdot \boldsymbol{n}=g & \text { on } \Gamma_{(f)},\end{cases}
$$

Keywords and phrases. Darcy's equations, spectral elements, a posteriori analysis.

1 Laboratoire de Mathématiques et de leurs Applications (U.M.R. 5142 CNRS), Bâtiment IPRA, Université de Pau et des Pays de l'Adour, avenue de l'Université, B.P. 1155, 64013 Pau Cedex, France. etienne.ahusborde@univ-pau.fr

2 Université de Bordeaux, I2M (UMR C.N.R.S. 5295), 33405 Talence, France. azaiez@ipb.fr

3 LMAC (E.A. 2222), Université de Technologie de Compiègne, B.P. 20529, 60205 Compiègne Cedex, France, and Université de Bordeaux, I2M (UMR C.N.R.S. 5295), 33405 Talence, France. faker.ben-belgacem@utc.fr

4 Laboratoire Jacques-Louis Lions, C.N.R.S. \& Université Pierre et Marie Curie, B.C. 187, 4 place Jussieu, 75252 Paris Cedex 05,

France. bernardi@ann.jussieu.fr 
where the unknowns are the velocity $\boldsymbol{u}$ and the pressure $p$ of the fluid. This system is an extension of Darcy's equations which model the flow of an incompressible viscous fluid in a saturated rigid porous medium, to the case where the pressure $p$ presents high variations. Indeed, in this case, it is no longer possible to neglect the dependence of the permeability of the medium, hence of the coefficient $\alpha$, with respect to $p$.

We refer to [3] for a first analysis of this nonlinear problem. The idea of this work relies on the following remark: The pressure presents high variations only on a part $\Omega_{\sharp}$ of the domain, so that in the remaining part $\Omega_{b}=\Omega \backslash \bar{\Omega}_{\sharp}$, replacing $\alpha(p)$ by a constant $\alpha_{0}$ does not induce a large modification of the solution. Thus, the iterative algorithm which is needed for handling the nonlinear term could be applied only on the subdomain $\Omega_{\sharp}$, which highly reduces the computational cost for the solution of any discretization of the problem. Moreover, as first explained in [12], a posteriori analysis leads to an automatic identification of the domains $\Omega_{b}$ and $\Omega_{\sharp}$. We refer to $[7,8]$ for the first application of this approach to fluid flows. We recall the existence of solutions to the full and simplified problems. The consistency of our approach follows from the a posteriori estimate between their solutions.

In a second step, we propose and study a spectral element discretization of the simplified problem. Such a discretization has been studied in [4] and more recently in [17] in the linear case of a constant coefficient $\alpha$. We perform the numerical analysis of this discretization in the nonlinear case and prove optimal a priori error estimates. Next, we perform the a posteriori analysis of both the simplification and the discretization. This leads us to a strategy for determining the subdomains $\Omega_{\mathrm{b}}$ and $\Omega_{\sharp}$ in order that the error issued from the simplification of the model is of the same order as the error issued from the discretization (see [7] for more details on a very similar strategy).

We propose an iterative algorithm for solving the nonlinear problem and prove its convergence for small variations of the function $\alpha$ (a similar algorithm is studied in [15] for a different problem). Only few recent works deal with the a posteriori analysis of iterative methods (see $[16,19]$ and the references therein). We perform this a posteriori analysis in the present case, the aim being of course to stop the iterative procedure when the error due to this algorithm is of the same order as the discretization error. Numerical experiments confirm the interest of the model when compared to the linear one and also the efficiency of our approach.

An outline of the paper is as follows.

- In Section 2, we present the variational formulation of problem (1.1) and recall its main properties. Next, we introduce a simplified problem and thereafter check the existence of a solution.

- Section 3 is devoted to the description and a priori analysis of the spectral element discretization.

- The a posteriori analysis of both the simplification and the discretization is performed in Section 4 . We also describe the strategy for the automatic choice of the subdomains $\Omega_{\mathrm{b}}$ and $\Omega_{\sharp}$.

- In Section 5, we propose an iterative algorithm for solving the nonlinear problem and prove its convergence together with optimal a posteriori estimates.

- Numerical experiments are presented in Section 6.

\section{The COMPLETE AND SIMPLIFIED MODELS}

From now on, we make the following assumptions:

(i) $\Gamma_{(p)}$ has a positive $(d-1)$-measure in $\partial \Omega$;

(ii) The function $\alpha$ is a continuous function from $\mathbb{R}$ into $\mathbb{R}$ and satisfies for two positive constants $\alpha_{1}$ and $\alpha_{2}$,

$$
\forall \xi \in \mathbb{R}, \quad \alpha_{1} \leq \alpha(\xi) \leq \alpha_{2} .
$$

Even if this is not true when the function $\alpha$ is exponential as suggested in [24], it does not seem restrictive to make this assumption (which is easily recovered by truncating the exponential), since in practical situations the pressure is always bounded. 
We consider the full scale of Sobolev spaces $H^{s}(\Omega), s \in \mathbb{R}$, and $W^{m, p}(\Omega), m \in \mathbb{N}, 1 \leq p \leq \infty$, equipped with the standard norms and seminorms. In order to write a variational formulation of problem (1.1), we introduce the space

$$
H_{(p)}^{1}(\Omega)=\left\{q \in H^{1}(\Omega) ; q=0 \text { on } \Gamma_{(p)}\right\} .
$$

Note that the traces of functions in $H_{(p)}^{1}(\Omega)$ on $\Gamma_{(f)}$ belong to $H_{00}^{\frac{1}{2}}\left(\Gamma_{(f)}\right)$ (see [21], Chap. 1, Sect. 11).

We recall from [11], Section XIII.1 that Darcy's equations even for a constant coefficient $\alpha$ admit several variational formulations. We have chosen here the formulation which enables us to treat the boundary condition on $p$ as an essential one and also seems the best adapted for handling the nonlinear term $\alpha(p) \boldsymbol{u}$ (see [1] for more numerical reasons). So, we consider the variational problem:

Find $(\boldsymbol{u}, p)$ in $L^{2}(\Omega)^{d} \times H^{1}(\Omega)$ such that

$$
p=p_{0} \quad \text { on } \Gamma_{(p)},
$$

and

$$
\begin{aligned}
& \forall \boldsymbol{v} \in L^{2}(\Omega)^{d}, \quad a^{[p]}(\boldsymbol{u}, \boldsymbol{v})+b(\boldsymbol{v}, p)=\int_{\Omega} \boldsymbol{f}(\boldsymbol{x}) \cdot \boldsymbol{v}(\boldsymbol{x}) \mathrm{d} \boldsymbol{x}, \\
& \forall q \in H_{(p)}^{1}(\Omega), \quad b(\boldsymbol{u}, q)=\langle g, q\rangle^{(f)},
\end{aligned}
$$

where the bilinear forms $a^{[\xi]}(\cdot, \cdot)$ for any measurable function $\xi$ on $\Omega$ and $b(\cdot, \cdot)$ are defined by

$$
a^{[\xi]}(\boldsymbol{u}, \boldsymbol{v})=\int_{\Omega} \alpha(\xi(\boldsymbol{x})) \boldsymbol{u}(\boldsymbol{x}) \cdot \boldsymbol{v}(\boldsymbol{x}) \mathrm{d} \boldsymbol{x}, \quad b(\boldsymbol{v}, q)=\int_{\Omega} \boldsymbol{v}(\boldsymbol{x}) \cdot(\operatorname{grad} q)(\boldsymbol{x}) \mathrm{d} \boldsymbol{x} .
$$

Here, $\langle\cdot, \cdot\rangle^{(f)}$ denotes the duality pairing between the dual space $H_{00}^{\frac{1}{2}}\left(\Gamma_{(f)}\right)^{\prime}$ and $H_{00}^{\frac{1}{2}}\left(\Gamma_{(f)}\right)$.

It is readily checked that the forms $a^{[\xi]}(\cdot, \cdot)$ and $b(\cdot, \cdot)$ are continuous on $L^{2}(\Omega)^{d} \times L^{2}(\Omega)^{d}$ and $L^{2}(\Omega)^{d} \times H^{1}(\Omega)$, respectively. Thus, some density arguments yield the equivalence of this problem with system (1.1).

Proposition 2.1. Assume that $\mathscr{D}\left(\Omega \cup \Gamma_{(f)}\right)$ is dense in $H_{(p)}^{1}(\Omega)$. For any data $\left(\boldsymbol{f}, p_{0}, g\right)$ in $L^{2}(\Omega)^{d} \times H^{\frac{1}{2}}\left(\Gamma_{(p)}\right) \times$ $H_{00}^{\frac{1}{2}}\left(\Gamma_{(f)}\right)^{\prime}$, problems (1.1) and (2.3)-(2.4) are equivalent, in the sense that any pair $(\boldsymbol{u}, p)$ in $L^{2}(\Omega)^{d} \times H^{1}(\Omega)$ is a solution of system (1.1) in the distribution sense if and only if it is a solution of problem (2.3)-(2.4).

The existence of a solution to problem (2.3)-(2.4) is established in [3], Theorem 2.3. Its proof relies on Brouwer's fixed point theorem, see e.g. [20], Chapter IV, Corollary 1.1, combined with the addition of a penalization term.

Theorem 2.2. For any data $\left(\boldsymbol{f}, p_{0}, g\right)$ in $L^{2}(\Omega)^{d} \times H^{\frac{1}{2}}\left(\Gamma_{(p)}\right) \times H_{00}^{\frac{1}{2}}\left(\Gamma_{(f)}\right)^{\prime}$, problem $(2.3)-(2.4)$ admits a solution $(\boldsymbol{u}, p)$ in $L^{2}(\Omega)^{d} \times H^{1}(\Omega)$. Moreover this solution satisfies

$$
\|\boldsymbol{u}\|_{L^{2}(\Omega)^{d}}+\|p\|_{H^{1}(\Omega)} \leq c\left(\|\boldsymbol{f}\|_{L^{2}(\Omega)^{d}}+\left\|p_{0}\right\|_{H^{\frac{1}{2}}\left(\Gamma_{(p)}\right)}+\|g\|_{H_{00}^{\frac{1}{2}\left(\Gamma_{(f)}\right)^{\prime}}}\right) .
$$

The uniqueness result is rather restrictive and has been discussed in [3], Proposition 2.4. It only holds for a smooth enough solution satisfying an appropriate condition. On the other hand, the following regularity property of the solution $(\boldsymbol{u}, p)$ is proved in [3], Proposition 2.5 thanks to the arguments in [22]. For the sake of generality, we state it in the general case of a curvilinear polygon or polyhedron (we refer to [18], Def. 2.2 for the exact definition of these domains, which of course includes standard polygons and polyhedra).

Proposition 2.3. If $\Omega$ is a curvilinear polygon or polyhedron, there exists a real number $\rho_{*}>2$ only depending on the geometry of $\Omega$ and on the ratio $\alpha_{1} / \alpha_{2}$ such that, for all $\rho, 2<\rho \leq \rho_{*}$, and for all data $\left(\boldsymbol{f}, p_{0}, g\right)$ in $L^{\rho}(\Omega)^{d} \times W^{1-\frac{1}{\rho}, \rho}\left(\Gamma_{(p)}\right) \times W^{-\frac{1}{\rho}, \rho}\left(\Gamma_{(f)}\right)$, any solution $(\boldsymbol{u}, p)$ of problem $(2.3)-(2.4)$ belongs to $L^{\rho}(\Omega)^{d} \times W^{1, \rho}(\Omega)$. 
To go further, we introduce a partition of $\Omega$ without overlap:

$$
\bar{\Omega}=\bar{\Omega}_{\sharp} \cup \bar{\Omega}_{b}, \quad \Omega_{\sharp} \cap \Omega_{b}=\emptyset .
$$

Next, we fix a constant $\alpha_{0}$ which satisfies

$$
\alpha_{1} \leq \alpha_{0} \leq \alpha_{2} .
$$

We thus define the function $\alpha^{*}$ on $\Omega \times \mathbb{R}$ by

$$
\forall \xi \in \mathbb{R}, \quad \alpha^{*}(\boldsymbol{x}, \xi)= \begin{cases}\alpha(\xi) & \text { for } \boldsymbol{x} \text { a.e. in } \Omega_{\sharp}, \\ \alpha_{0} & \text { for } \boldsymbol{x} \text { a.e. in } \Omega_{b} .\end{cases}
$$

Replacing $\alpha$ by $\alpha^{*}$ in system (1.1) (we do not write the corresponding problem for brevity) leads to the following equivalent variational problem:

Find $\left(\boldsymbol{u}^{*}, p^{*}\right)$ in $L^{2}(\Omega)^{d} \times H^{1}(\Omega)$ such that

$$
p^{*}=p_{0} \quad \text { on } \Gamma_{(p)},
$$

and

$$
\begin{aligned}
& \forall \boldsymbol{v} \in L^{2}(\Omega)^{d}, \quad a^{*\left[p^{*}\right]}\left(\boldsymbol{u}^{*}, \boldsymbol{v}\right)+b\left(\boldsymbol{v}, p^{*}\right)=\int_{\Omega} \boldsymbol{f}(\boldsymbol{x}) \cdot \boldsymbol{v}(\boldsymbol{x}) \mathrm{d} \boldsymbol{x}, \\
& \forall q \in H_{(p)}^{1}(\Omega), \quad b\left(\boldsymbol{u}^{*}, q\right)=\langle g, q\rangle^{(f)},
\end{aligned}
$$

where the bilinear form $a^{*[\xi]}(\cdot, \cdot)$ for any measurable function $\xi$ on $\Omega$ is now defined by

$$
a^{*[\xi]}(\boldsymbol{u}, \boldsymbol{v})=\int_{\Omega} \alpha^{*}(\boldsymbol{x}, \xi(\boldsymbol{x})) \boldsymbol{u}(\boldsymbol{x}) \cdot \boldsymbol{v}(\boldsymbol{x}) \mathrm{d} \boldsymbol{x} .
$$

It must be noted that the function $\alpha^{*}$ satisfies the same properties as $\alpha$, in particular (2.1). So, proving the next statement relies on exactly the same arguments as for Theorem 2.2 and Proposition 2.3.

Theorem 2.4. For any data $\left(\boldsymbol{f}, p_{0}, g\right)$ in $L^{2}(\Omega)^{d} \times H^{\frac{1}{2}}\left(\Gamma_{(p)}\right) \times H_{00}^{\frac{1}{2}}\left(\Gamma_{(f)}\right)^{\prime}$, problem $(2.10)-(2.11)$ admits a solution $\left(\boldsymbol{u}^{*}, p^{*}\right)$ in $L^{2}(\Omega)^{d} \times H^{1}(\Omega)$. Moreover this solution still satisfies (2.6) and, if $\Omega$ is a curvilinear polygon or polyhedron, the statement of Proposition 2.3 still holds for this solution, for the same value of $\rho_{0}$.

The links between the solutions $(\boldsymbol{u}, p)$ of problem $(2.3)-(2.4)$ and $\left(\boldsymbol{u}^{*}, p^{*}\right)$ of problem $(2.10)-(2.11)$ are brought to light in Section 4.

\section{THE DISCRETE PROBLEM AND ITS A PRIORI ANALYSIS}

As standard for spectral element methods, we consider a partition of $\Omega$ without overlap into a finite number of rectangles $(d=2)$ or rectangular parallelepipeds $(d=3)$ with edges parallel to the coordinate axes:

$$
\bar{\Omega}=\bigcup_{k=1}^{K} \bar{\Omega}_{k} \quad \text { and } \quad \Omega_{k} \cap \Omega_{k^{\prime}}=\emptyset, \quad 1 \leq k<k^{\prime} \leq K .
$$

We assume that

(i) both $\bar{\Gamma}_{(p)}$ and $\bar{\Gamma}_{(f)}$ are the union of whole edges $(d=2)$ or faces $(d=3)$ of elements $\Omega_{k}$,

(ii) the intersection of the boundaries of two subdomains, if not empty, is a vertex, a whole edge or a whole face $(d=3)$ of both elements (otherwise, the discretization would involve the mortar method as first proposed in [2] for this type of equation; we prefer to avoid this further complexity here).

We also make the hypothesis that each $\Omega_{k}$ is contained either in $\Omega_{b}$ or in $\Omega_{\sharp}$, which is in full agreement with our adaptivity process, see Section 4 . Note however that, as not standard, the decomposition can change according to the adaptivity process, so that we must be cautious with the dependency of the constants with respect to the decomposition. 
Let $N$ be a fixed positive integer. We introduce the discrete spaces

$$
\begin{aligned}
\mathbb{X}_{N}=\left\{v_{N} \in L^{2}(\Omega)^{d} ;\left.v_{N}\right|_{\Omega_{k}} \in \mathbb{P}_{N}\left(\Omega_{k}\right)^{d}, 1 \leq k \leq K\right\}, \\
\mathbb{M}_{N}=\left\{q_{N} \in H^{1}(\Omega) ;\left.q_{N}\right|_{\Omega_{k}} \in \mathbb{P}_{N}\left(\Omega_{k}\right), 1 \leq k \leq K\right\},
\end{aligned}
$$

where, for each nonnegative integer $n, \mathbb{P}_{n}\left(\Omega_{k}\right)$ stands for the space of restrictions to $\Omega_{k}$ of polynomials with $d$ variables and degree with respect to each variable $\leq n$. In view of the essential boundary conditions in problem $(2.3)-(2.4)$, we also consider the space

$$
\mathbb{M}_{N}^{(p)}=\mathbb{M}_{N} \cap H_{(p)}^{1}(\Omega) .
$$

We recall that there exist a unique set of $N+1$ nodes $\xi_{j}, 0 \leq j \leq N$, with $\xi_{0}=-1$ and $\xi_{N}=1$, and a unique set of $N+1$ weights $\rho_{j}, 0 \leq j \leq N$, such that the following equality holds

$$
\forall \Phi \in \mathbb{P}_{2 N-1}(-1,1), \quad \int_{-1}^{1} \Phi(\zeta) \mathrm{d} \zeta=\sum_{j=0}^{N} \Phi\left(\xi_{j}\right) \rho_{j}
$$

with obvious notation for the polynomial spaces $\mathbb{P}_{n}(-1,1)$. Moreover, the $\rho_{j}$ are positive. Denoting by $F_{k}$ one of the affine mappings that send the square or cube $]-1,1\left[^{d}\right.$ onto $\Omega_{k}$, we define a discrete product on all continuous functions $u$ and $v$ on $\bar{\Omega}_{k}$ by

$$
(u, v)_{N}^{k}=\left\{\begin{array}{lr}
\frac{\operatorname{meas}\left(\Omega_{k}\right)}{4} \sum_{i=0}^{N} \sum_{j=0}^{N} u \circ F_{k}\left(\xi_{i}, \xi_{j}\right) v \circ F_{k}\left(\xi_{i}, \xi_{j}\right) \rho_{i} \rho_{j} & \text { if } d=2, \\
\frac{\operatorname{meas}\left(\Omega_{k}\right)}{8} \sum_{i=0}^{N} \sum_{j=0}^{N} \sum_{m=0}^{N} u \circ F_{k}\left(\xi_{i}, \xi_{j}, \xi_{m}\right) v \circ F_{k}\left(\xi_{i}, \xi_{j}, \xi_{m}\right) \rho_{i} \rho_{j} \rho_{m} & \text { if } d=3,
\end{array}\right.
$$

next a global discrete product

$$
((u, v))_{N}=\sum_{k=1}^{K}(u, v)_{N}^{k} .
$$

It is readily checked that the Lagrange interpolation operator $\mathcal{I}_{N}$ at all nodes $F_{k}\left(\xi_{i}, \xi_{j}\right)$ or $F_{k}\left(\xi_{i}, \xi_{j}, \xi_{m}\right)$ maps continuous functions onto $\mathbb{M}_{N}$.

Similarly, on each edge or face $\Gamma_{\ell}$ of the $\Omega_{k}$, assuming for instance that the mapping $F_{k}$ maps $\left.\{-1\} \times\right]-1,1\left[{ }^{d-1}\right.$ onto $\Gamma_{\ell}$, we define a discrete product by

$$
(u, v)_{N}^{\Gamma_{\ell}}= \begin{cases}\frac{\operatorname{meas}\left(\Gamma_{\ell}\right)}{2} \sum_{j=0}^{N} u \circ F_{k}\left(\xi_{0}, \xi_{j}\right) v \circ F_{k}\left(\xi_{0}, \xi_{j}\right) \rho_{j} & \text { if } d=2, \\ \frac{\operatorname{meas}\left(\Gamma_{\ell}\right)}{4} \sum_{j=0}^{N} \sum_{m=0}^{N} u \circ F_{k}\left(\xi_{0}, \xi_{j}, \xi_{m}\right) v \circ F_{k}\left(\xi_{0}, \xi_{j}, \xi_{m}\right) \rho_{j} \rho_{m} & \text { if } d=3 .\end{cases}
$$

A global product on $\Gamma_{(f)}$ is then defined by

$$
((u, v))_{N}^{(f)}=\sum_{\ell \in \mathcal{L}_{(f)}}(u, v)_{N}^{\Gamma_{\ell}}
$$

where $\mathcal{L}_{(f)}$ stands for the set of indices $\ell$ such that $\Gamma_{\ell}$ is contained in $\Gamma_{(f)}$.

Finally, we introduce an approximation $p_{0 N}$ of the boundary datum $p_{0}$ : Assuming that $p_{0}$ is continuous on $\bar{\Gamma}_{(p)}$, for each edge $(d=2)$ or face $(d=3) \Gamma_{\ell}$ of an element $\Omega_{k}$ which is contained in $\Gamma_{(p)},\left.p_{0 N}\right|_{\Gamma_{\ell}}$ belongs to $\mathbb{P}_{N}\left(\Gamma_{\ell}\right)$ and is equal to $p_{0}$ at the $(N+1)^{d-1}$ nodes $F_{k}\left(\xi_{i}, \xi_{j}\right)$ or $F_{k}\left(\xi_{i}, \xi_{j}, \xi_{m}\right)$ which are located on $\bar{\Gamma}_{\ell}$. We denote by $i_{N}^{(p)}$ the corresponding interpolation operator.

We are thus in a position to write the discrete problem which is constructed from $(2.10)-(2.11)$ by the Galerkin method with numerical integration. Assuming that all data $\boldsymbol{f}, p_{0}$ and $g$ are continuous where needed, it reads 
Find $\left(\boldsymbol{u}_{N}, p_{N}\right)$ in $\mathbb{X}_{N} \times \mathbb{M}_{N}$ such that

$$
p_{N}=p_{0 N} \quad \text { on } \Gamma_{(p)},
$$

and

$$
\begin{aligned}
& \forall \boldsymbol{v}_{N} \in \mathbb{X}_{N}, \quad a_{N}^{*\left[p_{N}\right]}\left(\boldsymbol{u}_{N}, \boldsymbol{v}_{N}\right)+b_{N}\left(\boldsymbol{v}_{N}, p_{N}\right)=\left(\left(\boldsymbol{f}, \boldsymbol{v}_{N}\right)\right)_{N}, \\
& \forall q_{N} \in \mathbb{M}_{N}^{(p)}, \quad b_{N}\left(\boldsymbol{u}_{N}, q_{N}\right)=\left(\left(g, q_{N}\right)\right)_{N}^{(f)},
\end{aligned}
$$

where the bilinear forms $a_{N}^{*[\xi]}(\cdot, \cdot)$ for any continuous function $\xi$ on $\bar{\Omega}$ and $b_{N}(\cdot, \cdot)$ are defined by

$$
a_{N}^{*[\xi]}(\boldsymbol{u}, \boldsymbol{v})=\left(\left(\alpha^{*}(\cdot, \xi) \boldsymbol{u}, \boldsymbol{v}\right)\right)_{N}, \quad b_{N}(\boldsymbol{v}, q)=((\boldsymbol{v}, \operatorname{grad} q))_{N} .
$$

The existence of a solution to problem (3.5) -(3.6) can be established thanks to the same arguments as for the previous problems, i.e. by applying Brouwer's fixed point theorem. However we prefer to prove directly a more precise result by using the approach of Brezzi, Rappaz and Raviart [14], which also leads to a priori error estimates. This requires a modified (and shorter) formulation of both the continuous and discrete problems. From now on, we set:

$$
\mathcal{Z}(\Omega)=L^{2}(\Omega)^{d} \times H^{1}(\Omega), \quad \mathcal{Z}_{N}=\mathbb{X}_{N} \times \mathbb{M}_{N} .
$$

We thus introduce the Darcy operator with coefficient $\alpha_{0}$, namely the operator $\mathcal{T}$ which associates with any data $\left(\boldsymbol{f}, p_{0}, g\right)$ in $L^{2}(\Omega)^{d} \times H^{\frac{1}{2}}\left(\Gamma_{(p)}\right) \times H_{00}^{\frac{1}{2}}\left(\Gamma_{(f)}\right)^{\prime}$, the pair $(\boldsymbol{w}, r)$ in $\mathcal{Z}(\Omega)$ satisfying

$$
r=p_{0} \quad \text { on } \Gamma_{(p)},
$$

and

$$
\begin{aligned}
& \forall \boldsymbol{v} \in L^{2}(\Omega)^{d}, \quad a_{0}(\boldsymbol{w}, \boldsymbol{v})+b(\boldsymbol{v}, r)=\int_{\Omega} \boldsymbol{f}(\boldsymbol{x}) \cdot \boldsymbol{v}(\boldsymbol{x}) \mathrm{d} \boldsymbol{x}, \\
& \forall q \in H_{(p)}^{1}(\Omega), \quad b(\boldsymbol{w}, q)=\langle g, q\rangle^{(f)},
\end{aligned}
$$

where the bilinear form $a_{0}(\cdot, \cdot)$ is defined by

$$
a_{0}(\boldsymbol{w}, \boldsymbol{v})=\alpha_{0} \int_{\Omega} \boldsymbol{w}(\boldsymbol{x}) \cdot \boldsymbol{v}(\boldsymbol{x}) \mathrm{d} \boldsymbol{x} .
$$

Thus, problem (2.10)-(2.11) can equivalently be written as

$$
\mathcal{F}\left(U^{*}\right)=U^{*}-\mathcal{T} \mathcal{G}\left(U^{*}\right)=0, \quad \text { with } \quad \mathcal{G}(U)=\left(\boldsymbol{f}-\left(\alpha^{*}(\cdot, p)-\alpha_{0}\right) \boldsymbol{u}, p_{0}, g\right),
$$

with the notation $U^{*}=\left(\boldsymbol{u}^{*}, p^{*}\right)$.

Similarly, we introduce the operator $\mathcal{T}_{N}$ which associates with any data $\left(\boldsymbol{f}, p_{0}, g\right)$ in $L^{2}(\Omega)^{d} \times \mathscr{C}^{0}\left(\bar{\Gamma}_{(p)}\right) \times$ $H_{00}^{\frac{1}{2}}\left(\Gamma_{(f)}\right)^{\prime}$, the pair $\left(\boldsymbol{w}_{N}, r_{N}\right)$ in $\mathcal{Z}_{N}$ satisfying

$$
r_{N}=i_{N}^{(p)} p_{0} \quad \text { on } \Gamma_{(p)},
$$

and

$$
\begin{aligned}
& \forall \boldsymbol{v}_{N} \in \mathbb{X}_{N}, \quad a_{0 N}\left(\boldsymbol{w}_{N}, \boldsymbol{v}_{N}\right)+b_{N}\left(\boldsymbol{v}_{N}, r_{N}\right)=\int_{\Omega} \boldsymbol{f}(\boldsymbol{x}) \cdot \boldsymbol{v}_{N}(\boldsymbol{x}) \mathrm{d} \boldsymbol{x}, \\
& \forall q_{N} \in \mathbb{M}_{N}^{(p)}, \quad b_{N}\left(\boldsymbol{w}_{N}, q_{N}\right)=\left\langle g, q_{N}\right\rangle^{(f)},
\end{aligned}
$$

where the bilinear form $a_{0 N}(\cdot, \cdot)$ is defined on piecewise continuous functions $\boldsymbol{u}$ and $\boldsymbol{w}$ by

$$
a_{0 N}(\boldsymbol{w}, \boldsymbol{v})=\alpha_{0}((\boldsymbol{w}, \boldsymbol{v}))_{N} .
$$


So, problem (3.5)-(3.6) can be written as

$$
\mathcal{F}_{N}\left(U_{N}\right)=U_{N}-\mathcal{T}_{N} \mathcal{G}_{N}\left(U_{N}\right)=0, \quad \text { with } \quad \mathcal{G}_{N}\left(U_{N}\right)=\left(\boldsymbol{H}_{N}, p_{0}, K_{N}\right),
$$

with the notation $U_{N}=\left(\boldsymbol{u}_{N}, p_{N}\right)$, the first and third components $\boldsymbol{H}_{N}$ and $K_{N}$ being defined in the dual spaces of $\mathbb{X}_{N}$ and $\mathbb{M}_{N}^{(p)}$, respectively, by

$$
\begin{gathered}
\forall \boldsymbol{v}_{N} \in \mathbb{X}_{N}, \quad \int_{\Omega} \boldsymbol{H}_{N}(\boldsymbol{x}) \cdot \boldsymbol{v}_{N}(\boldsymbol{x}) \mathrm{d} \boldsymbol{x}=\left(\left(\boldsymbol{f}, \boldsymbol{v}_{N}\right)\right)_{N}-\left(\left(\left(\alpha^{*}\left(\cdot, p_{N}\right)-\alpha_{0}\right) \boldsymbol{u}_{N}, \boldsymbol{v}_{N}\right)\right)_{N}, \\
\forall q_{N} \in \mathbb{M}_{N}^{(p)}, \quad\left\langle K_{N}, q_{N}\right\rangle^{(f)}=\left(\left(g, q_{N}\right)\right)_{N}^{(f)} .
\end{gathered}
$$

We now work with the formulations (3.11) and (3.14). We observe that a pair $W=(\boldsymbol{w}, r)$ satisfies

$$
D \mathcal{F}(U) \cdot W=\mathcal{T}\left(\boldsymbol{h}, r_{0}, k\right)
$$

if and only if it is a solution in $L^{2}(\Omega)^{d} \times H^{1}(\Omega)$ of the problem:

$$
r=r_{0} \quad \text { on } \Gamma_{(p)},
$$

and

$$
\begin{aligned}
\forall \boldsymbol{v} \in L^{2}(\Omega)^{d}, \quad a^{*[p]}(\boldsymbol{w}, \boldsymbol{v})+\int_{\Omega} \partial_{\xi} \alpha^{*}(\boldsymbol{x}, p(\boldsymbol{x})) r(\boldsymbol{x}) \boldsymbol{u}(\boldsymbol{x}) \cdot \boldsymbol{v}(\boldsymbol{x}) \mathrm{d} \boldsymbol{x} & +b(\boldsymbol{v}, r) \\
& =\int_{\Omega} \boldsymbol{h}(\boldsymbol{x}) \cdot \boldsymbol{v}(\boldsymbol{x}) \mathrm{d} \boldsymbol{x},
\end{aligned}
$$

$\forall q \in H_{(p)}^{1}(\Omega), \quad b(\boldsymbol{w}, q)=\langle k, q\rangle^{(f)}$.

Of course this problem makes sense only if the integral in the first line is finite. So, from now on, we assume that the coefficient $\alpha$ belongs to $W^{1, \infty}(\mathbb{R})$ (further regularity will be required later on) and we work with a solution $\left(\boldsymbol{u}^{*}, p^{*}\right)$ which satisfies the following condition.

Assumption 3.1. The solution $U^{*}=\left(\boldsymbol{u}^{*}, p^{*}\right)$ of problem $(2.10)-(2.11)$

(i) belongs to $H^{s}(\Omega)^{d} \times H^{s+1}(\Omega)$ for some $s>0$ in dimension $d=2$ and $s>1$ in dimension $d=3$;

(ii) is such that $D \mathcal{F}\left(U^{*}\right)$ is an isomorphism of $L^{2}(\Omega)^{d} \times H^{1}(\Omega)$.

The existence of a solution $U^{*}$ satisfying part (i) of this assumption can be proved by the same arguments as for Theorem 2.4 in dimension $d=2$ but is not very likely in dimension $d=3$. On the other hand, part (ii) of this assumption is much weaker than the global uniqueness of the solution since it only yields its local uniqueness. We now state and prove two properties of the operator $\mathcal{T}_{N}$ that we need later on.

Lemma 3.2. The following stability property holds

$$
\left\|\mathcal{T}_{N}(\boldsymbol{f}, 0,0)\right\|_{\mathcal{Z}(\Omega)} \leq c \sup _{\boldsymbol{v}_{N} \in \mathbb{X}_{N}} \frac{\int_{\Omega} \boldsymbol{f}(\boldsymbol{x}) \cdot \boldsymbol{v}_{N}(\boldsymbol{x}) \mathrm{d} \boldsymbol{x}}{\left\|\boldsymbol{v}_{N}\right\|_{L^{2}(\Omega)^{d}}} .
$$

Proof. Setting $\left(\boldsymbol{w}_{N}, r_{N}\right)=\mathcal{T}_{N}(\boldsymbol{f}, 0,0)$, taking $\boldsymbol{v}_{N}$ equal to $\boldsymbol{w}_{N}$ in (3.13) and using the ellipticity of the form $a_{0 N}(\cdot, \cdot)$ on $\mathbb{X}_{N}$ with ellipticity constant $\alpha_{0}$ (see [11], Chap. IV, Cor. 1.10), we obtain the desired estimate for $\left\|\boldsymbol{w}_{N}\right\|_{L^{2}(\Omega)^{d}}$. On the other hand, the following inf-sup condition is easily derived by taking $\boldsymbol{v}_{N}$ equal to $\operatorname{grad} q_{N}$ and using the Poincaré-Friedrichs inequality on $H_{(p)}^{1}(\Omega)$ : There exists a constant $\beta>0$ independent of $N$ such that

$$
\forall q_{N} \in \mathbb{M}_{N}^{(p)}, q_{N} \neq 0, \quad \sup _{\boldsymbol{v}_{N} \in \mathbb{X}_{N}} \frac{b_{N}\left(\boldsymbol{v}_{N}, q_{N}\right)}{\left\|\boldsymbol{v}_{N}\right\|_{L^{2}(\Omega)^{d}}} \geq \beta\left\|q_{N}\right\|_{H^{1}(\Omega)} .
$$

Using this condition in the first line of (3.13) gives the estimate for $\left\|r_{N}\right\|_{H^{1}(\Omega)}$. 
Lemma 3.3. Let $\sigma$ and $s$ be real numbers, $\sigma>\frac{d-1}{2}, s>0$. The following convergence property holds for all $\left(\boldsymbol{f}, p_{0}, g\right)$ in $L^{2}(\Omega)^{d} \times H^{\sigma+\frac{1}{2}}\left(\Gamma_{(p)}\right) \times H_{00}^{\frac{1}{2}}\left(\Gamma_{(f)}\right)^{\prime}$ such that $\mathcal{T}\left(\boldsymbol{f}, p_{0}, g\right)$ belongs to $H^{s}(\Omega)^{d} \times H^{s+1}(\Omega)$

$$
\left\|\left(\mathcal{T}-\mathcal{T}_{N}\right)\left(\boldsymbol{f}, p_{0}, g\right)\right\|_{\mathcal{Z}(\Omega)} \leq c\left(N^{-s}\left\|\mathcal{T}\left(\boldsymbol{f}, p_{0}, g\right)\right\|_{H^{s}(\Omega)^{d} \times H^{s+1}(\Omega)}+N^{-\sigma}\left\|p_{0}\right\|_{H^{\sigma+\frac{1}{2}}\left(\Gamma_{(p)}\right)}\right) .
$$

Proof. In the simpler case $p_{0}=0$, the estimate is derived from the ellipticity of $a_{0 N}(\cdot, \cdot)$ and the inf-sup condition (3.18) by very standard arguments. In the general case, we introduce a stable lifting $\bar{p}_{0}$ of $p_{0}$ in $H^{\sigma+1}(\Omega)$ and observe that its Lagrange interpolant at all nodes $F_{k}\left(\xi_{i}, \xi_{j}\right)$ or $F_{k}\left(\xi_{i}, \xi_{j}, \xi_{m}\right)$ belongs to $\mathbb{M}_{N}$ and has its trace on $\Gamma_{(p)}$ equal to $p_{0 N}$. Adding and subtracting these liftings in (3.10) and (3.13) and using the same arguments as previously combined with the approximation properties of the Lagrange interpolation operator ([11], Chap. IV, Thm. 2.7) lead to the desired result.

A direct consequence of (3.17) and (3.19) is that, for any $\boldsymbol{f}$ in $L^{2}(\Omega)^{d}$,

$$
\lim _{N \rightarrow+\infty}\left\|\left(\mathcal{T}-\mathcal{T}_{N}\right)(\boldsymbol{f}, 0,0)\right\|_{\mathcal{Z}(\Omega)}=0 .
$$

We are thus in a position to prove the preliminary results which we need for applying the theorem of Brezzi, Rappaz and Raviart [14]. This requires an approximation $V_{N}^{*}=\left(\boldsymbol{v}_{N}^{*}, q_{N}^{*}\right)$ of $U^{*}$ in $\mathbb{X}_{N} \times \mathbb{M}_{N}$ which satisfies (see [11], Chap. III, Th. 2.4 and Chap. VI, Lem. 2.5) for the real number $s$ of Assumption 3.1 and $0 \leq t \leq s$,

$$
\begin{aligned}
& \left\|\boldsymbol{u}^{*}-\boldsymbol{v}_{N}^{*}\right\|_{H^{t}(\Omega)^{d}} \leq c N^{t-s}\left\|\boldsymbol{u}^{*}\right\|_{H^{s}(\Omega)^{d}}, \\
& \left\|p^{*}-q_{N}^{*}\right\|_{H^{t+1}(\Omega)} \leq c N^{t-s}\left\|p^{*}\right\|_{H^{s+1}(\Omega)} .
\end{aligned}
$$

We denote by $\mathcal{E}$ the space of endomorphisms of $\mathcal{Z}(\Omega)$.

Lemma 3.4. If the coefficient $\alpha$ is of class $\mathscr{C}^{2}$ on $\mathbb{R}$ with bounded derivatives and Assumption 3.1 holds, there exists a positive integer $N_{0}$ such that, for all $N \geq N_{0}$, the operator $D \mathcal{F}_{N}\left(V_{N}^{*}\right)$ is an isomorphism of $\mathbb{X}_{N} \times \mathbb{M}_{N}$, with the norm of its inverse bounded independently of $N$.

Proof. We use the expansion

$$
\begin{aligned}
D \mathcal{F}_{N}\left(V_{N}^{*}\right)=D \mathcal{F}\left(U^{*}\right) & +\left(\mathcal{T}-\mathcal{T}_{N}\right) D \mathcal{G}\left(U^{*}\right) \\
+ & \mathcal{T}_{N}\left(D \mathcal{G}\left(U^{*}\right)-D \mathcal{G}\left(V_{N}^{*}\right)\right)+\mathcal{T}_{N}\left(D \mathcal{G}\left(V_{N}^{*}\right)-D \mathcal{G}_{N}\left(V_{N}^{*}\right)\right) .
\end{aligned}
$$

Due to part (ii) of Assumption 3.1, it suffices to check that the last three terms in the right-hand side tend to zero when $N$ tends to $+\infty$. Let $W_{N}=\left(\boldsymbol{w}_{N}, r_{N}\right)$ be any element in the unit sphere of $\mathcal{Z}_{N}$.

1) We observe that

$$
D \mathcal{G}\left(U^{*}\right) \cdot W_{N}=\left(-\left(\alpha^{*}\left(\cdot, p^{*}\right)-\alpha_{0}\right) \boldsymbol{w}_{N}-\partial_{\xi} \alpha^{*}\left(\cdot, p^{*}\right) r_{N} \boldsymbol{u}^{*}, 0,0\right) .
$$

By combining Assumption 3.1 with [13], Theorem 1', we observe that the term $\alpha^{*}\left(\cdot, p^{*}\right)-\alpha_{0}$ also belongs to $H^{\min \{s, 1\}+1}(\Omega)$, hence to a compact subset of $L^{\infty}(\Omega)$. Since $\boldsymbol{w}_{N}$ is bounded in $L^{2}(\Omega)^{d},\left(\alpha^{*}\left(\cdot, p^{*}\right)-\alpha_{0}\right) \boldsymbol{w}_{N}$ belongs to a compact subset of $L^{2}(\Omega)^{d}$. Similarly, since Assumption 3.1 yields that $\boldsymbol{u}^{*}$ belongs to $L^{\rho}(\Omega)^{d}$, with $\rho>2$ in dimension $d=2$ and $\rho>3$ in dimension $d=3$, we use the compactness of the imbedding of $H^{1}(\Omega)$ into $L^{\rho^{\prime}}(\Omega)$, with $\frac{1}{\rho}+\frac{1}{\rho^{\prime}}=\frac{1}{2}$, to derive that the quantity $\partial_{\xi} \alpha^{*}\left(\cdot, p^{*}\right) r_{N} \boldsymbol{u}^{*}$ also belongs to a compact set of $L^{2}(\Omega)^{d}$. Thus, it follows from (3.17) and (3.20) that

$$
\lim _{N \rightarrow+\infty}\left\|\left(\mathcal{T}-\mathcal{T}_{N}\right) D \mathcal{G}\left(U^{*}\right)\right\|_{\mathcal{E}}=0 .
$$

2) Due to (3.17) and the definition of $D \mathcal{G}$, we must now investigate the convergence of the three terms

$$
\left(\alpha^{*}\left(\cdot, p^{*}\right)-\alpha^{*}\left(\cdot, q_{N}^{*}\right)\right) \boldsymbol{w}_{N}, \quad\left(\partial_{\xi} \alpha^{*}\left(\cdot, p^{*}\right)-\partial_{\xi} \alpha^{*}\left(\cdot, q_{N}^{*}\right)\right) r_{N} \boldsymbol{u}^{*}, \quad \partial_{\xi} \alpha^{*}\left(\cdot, q_{N}^{*}\right) r_{N}\left(\boldsymbol{u}^{*}-\boldsymbol{v}_{N}^{*}\right)
$$


in $L^{2}(\Omega)^{d}$. The convergence of the first one follows from the Lipschitz property of $\alpha^{*}$ and (3.21) applied with a $t<s$ such that $H^{t+1}(\Omega)$ is imbedded in $L^{\infty}(\Omega)$. From the Lipschitz property of $\partial_{\xi} \alpha^{*}$, since $r_{N} \boldsymbol{u}^{*}$ is bounded in $L^{2}(\Omega)^{d}$, the same choice of $t$ yields the convergence of the second term. Finally, the convergence of the third term results from (3.21) applied with a $t<s$ such that $H^{t}(\Omega)$ is imbedded in $L^{r}(\Omega)$, with $r>2$ in dimension $d=2$ and $r=3$ in dimension $d=3$. Combining all this yields

$$
\lim _{N \rightarrow+\infty}\left\|\mathcal{T}_{N}\left(D \mathcal{G}\left(U^{*}\right)-D \mathcal{G}\left(V_{N}^{*}\right)\right)\right\|_{\mathcal{E}}=0
$$

3) The last term comes from numerical integration, so that proving its convergence is rather technical. Using once more (3.17), we have to evaluate the quantities, for all $\boldsymbol{z}_{N}$ in the unit sphere of $\mathbb{X}_{N}$,

$$
\begin{array}{r}
a^{*\left[q_{N}^{*}\right]}\left(\boldsymbol{w}_{N}, \boldsymbol{z}_{N}\right)-a_{N}^{*\left[q_{N}^{*}\right]}\left(\boldsymbol{w}_{N}, \boldsymbol{z}_{N}\right), \quad a_{0}\left(\boldsymbol{w}_{N}, \boldsymbol{z}_{N}\right)-a_{0 N}\left(\boldsymbol{w}_{N}, \boldsymbol{z}_{N}\right) \\
\int_{\Omega} \partial_{\xi} \alpha^{*}\left(\cdot, q_{N}^{*}\right)(\boldsymbol{x}) r_{N}(\boldsymbol{x}) \boldsymbol{v}_{N}^{*}(\boldsymbol{x}) \cdot \boldsymbol{z}_{N}(\boldsymbol{x}) \mathrm{d} \boldsymbol{x}-\left(\left(\partial_{\xi} \alpha^{*}\left(\cdot, q_{N}^{*}\right) r_{N} \boldsymbol{v}_{N}^{*}, \boldsymbol{z}_{N}\right)\right)_{N}
\end{array}
$$

Since the arguments for evaluating the three of them are very similar, we only consider the third one which is the more complex. We denote it by $B_{N}$ for brevity. If $N^{\diamond}$ stands for the integer part of $\frac{N-1}{3}$, we introduce approximations $A_{N \diamond}$ of $\partial_{\xi} \alpha^{*}\left(\cdot, q_{N}^{*}\right)$ and $r_{N^{\diamond}}$ of $r_{N}$ in $\mathbb{M}_{N^{\diamond}}$, and also $\boldsymbol{v}_{N^{\diamond}}$ of $\boldsymbol{u}^{*}$ in $\mathbb{X}_{N^{\diamond}}$ (with obvious notation) and we note the identity

$$
\int_{\Omega} A_{N^{\diamond}}(\boldsymbol{x}) r_{N^{\diamond}}(\boldsymbol{x}) \boldsymbol{v}_{N^{\diamond}}(\boldsymbol{x}) \cdot \boldsymbol{z}_{N}(\boldsymbol{x}) \mathrm{d} \boldsymbol{x}=\left(\left(A_{N^{\diamond}} r_{N^{\diamond}} \boldsymbol{v}_{N^{\diamond}}, \boldsymbol{z}_{N}\right)\right)_{N} .
$$

Inserting it into the definition of $B_{N}$ and using [11], Chap. IV, Cor. 1.10), we obtain

$$
B_{N} \leq\left\|\partial_{\xi} \alpha^{*}\left(\cdot, q_{N}^{*}\right) r_{N} \boldsymbol{v}_{N}^{*}-A_{N^{\diamond}} r_{N^{\diamond}} \boldsymbol{v}_{N^{\diamond}}\right\|_{L^{2}(\Omega)^{d}}+\left\|\mathcal{I}_{N}\left(\mathcal{I}_{N}\left(\partial_{\xi} \alpha^{*}\left(\cdot, q_{N}^{*}\right)\right) r_{N} \boldsymbol{v}_{N}^{*}-A_{N^{\diamond}} r_{N^{\diamond}} \boldsymbol{v}_{N^{\diamond}}\right)\right\|_{L^{2}(\Omega)^{d}} .
$$

We recall from [10], Remark 13.5 the stability of the operator $\mathcal{I}_{N}$ on polynomials of degree $\leq 3 N$ in $L^{2}(\Omega)$. Thus, by using triangle inequalities, the imbedding of $H^{1}(\Omega)$ into any $L^{q}(\Omega)$ in dimension $d=2$, into $L^{6}(\Omega)$ in dimension $d=3$ and some stability properties of the polynomials $r_{N^{\diamond}}$ and $\boldsymbol{v}_{N^{\diamond}}$, it suffices to prove the convergence of

$$
\begin{array}{r}
\left\|\partial_{\xi} \alpha^{*}\left(\cdot, q_{N}^{*}\right)-\mathcal{I}_{N} \partial_{\xi} \alpha^{*}\left(\cdot, q_{N}^{*}\right)\right\|_{H^{1}(\Omega)}, \quad\left\|\partial_{\xi} \alpha^{*}\left(\cdot, q_{N}^{*}\right)-A_{N^{\diamond}}\right\|_{H^{1}(\Omega)}, \\
\left\|r_{N}-r_{N^{\diamond}}\right\|_{H^{1}(\Omega)}\left\|\boldsymbol{v}_{N}^{*}-\boldsymbol{v}_{N^{\diamond}}\right\|_{H^{t}(\Omega)^{d}},
\end{array}
$$

with $t>0$ in dimension $d=2, t=1$ in dimension $d=3$. This results from the properties of the operator $\mathcal{I}_{N}$ ([11], Thm. IV.2.7) and from appropriate choices of $A_{N^{\diamond}}, r_{N^{\diamond}}$, and $\boldsymbol{v}_{N^{\diamond}}$ ([11], Sect. III.2). Thus, we obtain

$$
\lim _{N \rightarrow+\infty} \| \mathcal{T}_{N}\left(D \mathcal{G}\left(V_{N}^{*}\right)-D \mathcal{G}_{N}\left(V_{N}^{*}\right) \|_{\mathcal{E}}=0\right.
$$

The desired result is now an easy consequence of (3.22), (3.23) and (3.24).

Lemma 3.5. If the coefficient $\alpha$ is of class $\mathscr{C}^{2}$ on $\mathbb{R}$ with bounded derivatives and Assumption 3.1 holds, there exist a neighbourhood of $V_{N}^{*}$ in $\mathcal{Z}_{N}$ and a constant $\lambda>0$ such that the operator $D \mathcal{F}_{N}$ satisfies the following Lipschitz property, for all $V_{N}$ in this neighbourhood,

$$
\left\|D \mathcal{F}_{N}\left(V_{N}^{*}\right)-D \mathcal{F}_{N}\left(V_{N}\right)\right\|_{\mathcal{E}} \leq \lambda \mu(N)\left\|V_{N}^{*}-V_{N}\right\|_{\mathcal{Z}(\Omega)},
$$

with $\mu(N)$ equal to $|\log N|^{\frac{1}{2}}$ in dimension $d=2$ and to $N$ in dimension $d=3$. 
Proof. We set: $V_{N}=\left(\boldsymbol{v}_{N}, q_{N}\right)$. Owing to (3.17), we have to evaluate the quantities, for any $W_{N}=\left(\boldsymbol{w}_{N}, r_{N}\right)$ in the unit sphere of $\mathcal{Z}_{N}$ and $\boldsymbol{z}_{N}$ in the unit sphere of $\mathbb{X}_{N}$,

$$
\begin{array}{r}
a_{N}^{*\left[q_{N}^{*}\right]}\left(\boldsymbol{w}_{N}, \boldsymbol{z}_{N}\right)-a_{N}^{*\left[q_{N}\right]}\left(\boldsymbol{w}_{N}, \boldsymbol{z}_{N}\right) \\
\quad \text { and } \quad\left(\left(\partial_{\xi} \alpha^{*}\left(\cdot, q_{N}^{*}\right) r_{N} \boldsymbol{v}_{N}^{*}, \boldsymbol{z}_{N}\right)\right)_{N}-\left(\left(\partial_{\xi} \alpha^{*}\left(\cdot, q_{N}\right) r_{N} \boldsymbol{v}_{N}, \boldsymbol{z}_{N}\right)\right)_{N} .
\end{array}
$$

For the same reasons as in the previous proof, we only consider the second one, that we denote by $C_{N}$. Next, we write

$$
\begin{aligned}
C_{N}=\left(\left(\partial_{\xi} \alpha^{*}\left(\cdot, q_{N}\right) r_{N}\left(\boldsymbol{v}_{N}^{*}-\boldsymbol{v}_{N}\right), \boldsymbol{z}_{N}\right)\right)_{N} & \\
& +\left(\left(\left(\partial_{\xi} \alpha^{*}\left(\cdot, q_{N}^{*}\right)-\partial_{\xi} \alpha^{*}\left(\cdot, q_{N}\right)\right) r_{N} \boldsymbol{v}_{N}^{*}, \boldsymbol{z}_{N}\right)\right)_{N},
\end{aligned}
$$

whence, by using once more ([11], Chap. IV, Cor. 1.10),

$$
\begin{aligned}
C_{N} \leq\left\|\partial_{\xi} \alpha^{*}\left(\cdot, q_{N}\right)\right\|_{L^{\infty}(\Omega)} \| \mathcal{I}_{N}\left(r_{N}\right. & \left.\left(\boldsymbol{v}_{N}^{*}-\boldsymbol{v}_{N}\right)\right) \|_{L^{2}(\Omega)^{d}} \\
& +\left\|\partial_{\xi} \alpha^{*}\left(\cdot, q_{N}^{*}\right)-\partial_{\xi} \alpha^{*}\left(\cdot, q_{N}\right)\right\|_{L^{\infty}(\Omega)}\left\|\mathcal{I}_{N}\left(r_{N} \boldsymbol{v}_{N}^{*}\right)\right\|_{L^{2}(\Omega)^{d}} .
\end{aligned}
$$

By combining the stability of the operator $\mathcal{I}_{N}$ on polynomials of degree $\leq 2 N$ [10], Remark 13.5 with the fact that $\partial_{\xi} \alpha^{*}$ is bounded and Lipschitz-continuous with a bounded Lipschitz constant, we derive that, for any $\rho>2$ and with $\frac{1}{\rho}+\frac{1}{\rho^{\prime}}=\frac{1}{2}$,

$$
\left|C_{N}\right| \leq c\left(\left\|r_{N}\right\|_{L^{\infty}(\Omega)}\left\|\boldsymbol{v}_{N}^{*}-\boldsymbol{v}_{N}\right\|_{L^{2}(\Omega)^{d}}+\left\|q_{N}^{*}-q_{N}\right\|_{L^{\infty}(\Omega)}\left\|r_{N}\right\|_{L^{\rho^{\prime}}(\Omega)}\left\|\boldsymbol{v}_{N}^{*}\right\|_{L^{\rho}(\Omega)^{d}}\right) .
$$

It can be noted from Assumption 3.1 and (3.21) that $\boldsymbol{v}_{N}^{*}$ is bounded in $L^{\rho}(\Omega)$, for some $\rho>2$ in dimension $d=2$ and $\rho=3$ in dimension $d=3$. Moreover, $H^{1}(\Omega)$ is imbedded into the corresponding space $L^{\rho^{\prime}}(\Omega)$. We conclude by applying the inverse inequality [9], Chapter III, Proposition 2.1, valid for any polynomial $\varphi_{N}$ in $\mathbb{P}_{N}\left(\Omega_{k}\right)$

$$
\left\|\varphi_{N}\right\|_{L^{\infty}\left(\Omega_{k}\right)} \leq c N^{\frac{2 d}{\delta}}\left\|\varphi_{N}\right\|_{L^{\delta}\left(\Omega_{k}\right)}
$$

and noting that

- in dimension $d=3, H^{1}\left(\Omega_{k}\right)$ is embedded in $L^{6}\left(\Omega_{k}\right)$,

- in dimension $d=2, H^{1}\left(\Omega_{k}\right)$ is embedded in any $L^{\delta}\left(\Omega_{k}\right)$ with norm of the imbedding smaller than $c \sqrt{\delta}$, see [25] (we thus take $\delta$ equal to $\log N$ ).

Lemma 3.6. If the coefficient $\alpha$ is of class $\mathscr{C}^{2}$ on $\mathbb{R}$ with bounded derivatives, Assumption 3.1 holds and the data $\left(\boldsymbol{f}, p_{0}, g\right)$ belong to $H^{\sigma}(\Omega)^{d} \times H^{\sigma+\frac{1}{2}}\left(\Gamma_{(p)}\right) \times H^{\sigma}\left(\Gamma_{(f)}\right), \sigma>\frac{d}{2}$, the following estimate is satisfied

$$
\begin{aligned}
\left\|\mathcal{F}_{N}\left(V_{N}^{*}\right)\right\|_{\mathcal{Z}(\Omega)} \leq c\left(N^{-s}\right. & \left(\left\|\boldsymbol{u}^{*}\right\|_{H^{s}(\Omega)^{d}}+\left\|p^{*}\right\|_{H^{s+1}(\Omega)}\right) \\
& \left.+N^{-\sigma}\left(\|\boldsymbol{f}\|_{H^{\sigma}(\Omega)^{d}}+\left\|p_{0}\right\|_{H^{\sigma+\frac{1}{2}\left(\Gamma_{(p)}\right)}}+\|g\|_{H^{\sigma}\left(\Gamma_{(f)}\right)}\right)\right) .
\end{aligned}
$$

Proof. Since $\mathcal{F}\left(U^{*}\right)$ is zero, we have

$$
\begin{aligned}
\left\|\mathcal{F}_{N}\left(V_{N}^{*}\right)\right\|_{\mathcal{Z}(\Omega)} \leq \| U^{*}- & V_{N}^{*}\left\|_{\mathcal{Z}(\Omega)}+\right\|\left(\mathcal{T}-\mathcal{T}_{N}\right) \mathcal{G}\left(U^{*}\right) \|_{\mathcal{Z}(\Omega)} \\
& +\left\|\mathcal{T}_{N}\left(\mathcal{G}\left(U^{*}\right)-\mathcal{G}\left(V_{N}^{*}\right)\right)\right\|_{\mathcal{Z}(\Omega)}+\left\|\mathcal{T}_{N}\left(\mathcal{G}\left(V_{N}^{*}\right)-\mathcal{G}_{N}\left(V_{N}^{*}\right)\right)\right\|_{\mathcal{Z}(\Omega)} .
\end{aligned}
$$

The first term is bounded in (3.21). Evaluating the second term follows from (3.19) by noting that $\mathcal{T} \mathcal{G}\left(U^{*}\right)$ is equal to $U^{*}$. To bound the third one, we apply (3.17) and note from the properties of the function $\alpha$ and Assumption 3.1 that

$$
\left\|\left(\alpha^{*}\left(\cdot, p^{*}\right)-\alpha_{0}\right) \boldsymbol{u}^{*}-\left(\alpha^{*}\left(\cdot, q_{N}^{*}\right)-\alpha_{0}\right) \boldsymbol{v}_{N}^{*}\right\|_{L^{2}(\Omega)^{d}} \leq c\left(\left\|\boldsymbol{u}^{*}-\boldsymbol{v}_{N}^{*}\right\|_{L^{2}(\Omega)^{d}}+\left\|p^{*}-q_{N}^{*}\right\|_{H^{1}(\Omega)}\right),
$$


so that the estimate follows from (3.21). Finally, proving the estimate for the fourth term is obtained by applying (3.17), using the standard arguments for the error issued from numerical integration (see [11], Sect. V.1 for instance) combined with the same arguments as in part 3) of the proof of Lemma 3.4 (i.e. introducing approximations of $\alpha^{*}\left(\cdot, q_{N}^{*}\right)$ in $\mathbb{M}_{N \diamond}$ and of $\boldsymbol{u}^{*}$ in $\mathbb{X}_{N^{\diamond}}$, where now $N^{\diamond}$ stands for the integer part of $\frac{N-1}{2}$ ).

Owing to Lemmas 3.4 to 3.6, we are in a position to apply the theorem of Brezzi, Rappaz and Raviart [14]. Note that it requires Assumption 3.1 since we need that, for the quantity $\mu(N)$ introduced in Lemma 3.5, $\lim _{N \rightarrow+\infty} \mu(N) N^{-s}=0$. A similar condition must hold on the data.

Theorem 3.7. If the coefficient $\alpha$ is of class $\mathscr{C}^{2}$ on $\mathbb{R}$ with bounded derivatives, Assumption 3.1 holds and the data $\left(\boldsymbol{f}, p_{0}, g\right)$ belong to $H^{\sigma}(\Omega)^{d} \times H^{\sigma+\frac{1}{2}}\left(\Gamma_{(p)}\right) \times H^{\sigma}\left(\Gamma_{(f)}\right), \sigma>\frac{d}{2}$, there exist a positive integer $N^{*}$ and a positive constant $\rho$ such that, for $N \geq N^{*}$, problem (3.5)-(3.6) has a unique solution $\left(\boldsymbol{u}_{N}, p_{N}\right)$ in the ball with centre $\left(\boldsymbol{u}^{*}, p^{*}\right)$ and radius $\rho \mu(N)^{-1}$. Moreover this solution satisfies the following a priori error estimate

$$
\begin{aligned}
& \left\|\boldsymbol{u}^{*}-\boldsymbol{u}_{N}\right\|_{L^{2}(\Omega)^{d}}+\left\|p^{*}-p_{N}\right\|_{H^{1}(\Omega)} \\
& \leq c\left(\boldsymbol{u}^{*}, p^{*}\right)\left(N^{-s}\left(\left\|\boldsymbol{u}^{*}\right\|_{H^{s}(\Omega)^{d}}+\left\|p^{*}\right\|_{H^{s+1}(\Omega)}\right)\right. \\
& \left.\quad+N^{-\sigma}\left(\|\boldsymbol{f}\|_{H^{\sigma}(\Omega)^{d}}+\left\|p_{0}\right\|_{H^{\sigma+\frac{1}{2}\left(\Gamma_{(p)}\right)}}+\|g\|_{H^{\sigma}\left(\Gamma_{(f)}\right)}\right)\right),
\end{aligned}
$$

where the constant $c\left(\boldsymbol{u}^{*}, p^{*}\right)$ only depends on the solution $\left(\boldsymbol{u}^{*}, p^{*}\right)$.

The assumptions for Theorem 3.7, more precisely part (i) of Assumption 3.1, are very likely in dimension $d=2$ but they are not really for a non convex domain in dimension $d=3$. However the convergence of the discretization when the data are continuous can be derived from the previous statement.

\section{A POSTERIORI ANALYSIS}

As now standard for multistep discretizations, the a posteriori analysis that we perform relies on the triangle inequalities

$$
\begin{aligned}
\left\|\boldsymbol{u}-\boldsymbol{u}_{N}\right\|_{L^{2}(\Omega)^{d}} \leq\left\|\boldsymbol{u}-\boldsymbol{u}^{*}\right\|_{L^{2}(\Omega)^{d}}+\left\|\boldsymbol{u}^{*}-\boldsymbol{u}_{N}\right\|_{L^{2}(\Omega)^{d}} \\
\left\|p-p_{N}\right\|_{H^{1}(\Omega)} \leq\left\|p-p^{*}\right\|_{H^{1}(\Omega)}+\left\|p^{*}-p_{N}\right\|_{H^{1}(\Omega)} .
\end{aligned}
$$

Indeed, we wish to uncouple as much as possible the errors issued from the simplification and the discretization. In both cases, proving an upper bound for these errors consists in applying the theorem of Pousin and Rappaz [23] (see also [26], Prop. 2.1 for a more precise version).

\subsection{Error due to the simplification of the model}

On each domain $\Omega_{k}, 1 \leq k \leq K$, we define the error indicator

$$
\eta_{N, k}^{(s)}=\left\|\left(\alpha\left(p_{N}\right)-\alpha^{*}\left(\cdot, p_{N}\right)\right) \boldsymbol{u}_{N}\right\|_{L^{2}\left(\Omega_{k}\right)^{d}} .
$$

It can be noted that all $\eta_{N, k}^{(s)}$ such that $\Omega_{k}$ is contained in $\Omega_{\sharp}$ are zero. Otherwise, they are given by

$$
\eta_{N, k}^{(s)}=\left\|\left(\alpha\left(p_{N}\right)-\alpha_{0}\right) \boldsymbol{u}_{N}\right\|_{L^{2}\left(\Omega_{k}\right)^{d}} .
$$

Remark 4.1. In practice, $\alpha\left(p_{N}\right)$ is most often replaced by $\mathcal{I}_{N} \alpha\left(p_{N}\right)$ in the previous definition (4.3), in order to make the $\eta_{N, k}^{(s)}$ easier to compute. The next analysis is still valid in this case. 
Using the notation introduced in Section 3, we observe that any solution $U=(\boldsymbol{u}, p)$ of problem $(2.3)-(2.4)$ satisfies

$$
\mathcal{F}_{0}(U)=U-\mathcal{T} \mathcal{G}_{0}(U)=0, \quad \text { with } \quad \mathcal{G}_{0}(U)=\left(\boldsymbol{f}-\left(\alpha(p)-\alpha_{0}\right) \boldsymbol{u}, p_{0}, g\right),
$$

while any solution $U^{*}=\left(\boldsymbol{u}^{*}, p^{*}\right)$ of problem (2.10)-(2.11) satisfies (3.11). Thus, we are led to make the following analogue of Assumption 3.1.

Assumption 4.2. The solution $U=(\boldsymbol{u}, p)$ of problem $(2.3)-(2.4)$

(i) belongs to $H^{s}(\Omega)^{d} \times H^{s+1}(\Omega)$ for some $s>0$ in dimension $d=2$ and $s>\frac{1}{2}$ in dimension $d=3$;

(ii) is such that $D \mathcal{F}_{0}(U)$ is an isomorphism of $L^{2}(\Omega)^{d} \times H^{1}(\Omega)$.

Indeed, this assumption is needed to prove the following lemma.

Lemma 4.3. If Assumption 4.2 holds, the mapping: $V \mapsto D \mathcal{F}_{0}(V)$ is continuous on $H^{s}(\Omega)^{d} \times H^{s+1}(\Omega)$ and Lipschitz-continuous on a neighbourhood of $U$ in this same space.

Proof. We only check the Lipschitz property. Let $V_{1}=\left(\boldsymbol{v}_{1}, q_{1}\right)$ and $V_{2}=\left(\boldsymbol{v}_{2}, q_{2}\right)$ be two elements in the neighbourhood of $U$. For the same reasons as in the proof of Lemma 3.5, we have to evaluate the quantities, for any $W=(\boldsymbol{w}, r)$ in the unit sphere of $\mathcal{Z}(\Omega)$ and $\boldsymbol{z}$ in the unit sphere of $L^{2}(\Omega)^{d}$,

$$
\begin{aligned}
& a^{\left[q_{1}\right]}(\boldsymbol{w}, \boldsymbol{z})-a^{\left[q_{2}\right]}(\boldsymbol{w}, \boldsymbol{z}) \\
& \text { and } \int_{\Omega} \partial_{\xi} \alpha\left(\boldsymbol{x}, q_{1}(\boldsymbol{x})\right) r(\boldsymbol{x}) \boldsymbol{v}_{1}(\boldsymbol{x}) \cdot \boldsymbol{z}(\boldsymbol{x}) \mathrm{d} \boldsymbol{x}-\int_{\Omega} \partial_{\xi} \alpha\left(\boldsymbol{x}, q_{2}(\boldsymbol{x})\right) r(\boldsymbol{x}) \boldsymbol{v}_{2}(\boldsymbol{x}) \cdot \boldsymbol{z}(\boldsymbol{x}) \mathrm{d} \boldsymbol{x} .
\end{aligned}
$$

Bounding the first term follows from the imbedding of $H^{s+1}(\Omega)$ into $L^{\infty}(\Omega)$. On the other hand, denoting by $C$ the second term, we have

$$
\begin{aligned}
C=\int_{\Omega}\left(\partial_{\xi} \alpha\left(\boldsymbol{x}, q_{1}(\boldsymbol{x})\right)-\partial_{\xi} \alpha(\boldsymbol{x},\right. & \left.\left.q_{2}(\boldsymbol{x})\right)\right) r(\boldsymbol{x}) \boldsymbol{v}_{1}(\boldsymbol{x}) \cdot \boldsymbol{z}(\boldsymbol{x}) \mathrm{d} \boldsymbol{x} \\
& +\int_{\Omega} \partial_{\xi} \alpha\left(\boldsymbol{x}, q_{2}(\boldsymbol{x})\right) r(\boldsymbol{x})\left(\boldsymbol{v}_{1}-\boldsymbol{v}_{2}\right)(\boldsymbol{x}) \cdot \boldsymbol{z}(\boldsymbol{x}) \mathrm{d} \boldsymbol{x},
\end{aligned}
$$

whence, with $\frac{1}{\rho}+\frac{1}{\rho^{\prime}}=\frac{1}{2}$,

$$
C \leq c\left(\left\|q_{1}-q_{2}\right\|_{L^{\infty}(\Omega)}\|r\|_{L^{\rho^{\prime}(\Omega)}}\left\|\boldsymbol{v}_{1}\right\|_{L^{\rho}(\Omega)^{d}}+\|r\|_{L^{\rho^{\prime}(\Omega)}}\left\|\boldsymbol{v}_{1}-\boldsymbol{v}_{2}\right\|_{L^{\rho}(\Omega)^{d}}\right) .
$$

Finally, we take $\rho>2$ in dimension 2 (small enough for $H^{s}(\Omega)$ to be imbedded in $L^{\rho}(\Omega)$ ) and $\rho=3$ in dimension 3. Thus it follows from Assumption 4.2 and the imbedding of $H^{1}(\Omega)$ in $L^{\rho^{\prime}}(\Omega)$ that all the terms in the previous inequality are bounded.

Proposition 4.4. If Assumption 4.2 holds, there exists a neighbourhood of $U$ in $H^{s}(\Omega)^{d} \times H^{s+1}(\Omega)$ such that the following a posteriori error estimate holds for any solution $U^{*}=\left(\boldsymbol{u}^{*}, p^{*}\right)$ of problem $(2.10)-(2.11)$ in this neighbourhood

$$
\left\|\boldsymbol{u}-\boldsymbol{u}^{*}\right\|_{L^{2}(\Omega)^{d}}+\left\|p-p^{*}\right\|_{H^{1}(\Omega)} \leq c(\boldsymbol{u}, p)\left(\left(\sum_{k=1}^{K}\left(\eta_{N, k}^{(s)}\right)^{2}\right)^{\frac{1}{2}}+\left\|\boldsymbol{u}^{*}-\boldsymbol{u}_{N}\right\|_{L^{2}(\Omega)^{d}}+\left\|p^{*}-p_{N}\right\|_{H^{1}(\Omega)}\right),
$$

where the constant $c(\boldsymbol{u}, p)$ only depends on the solution $U$. 
Proof. Owing to Lemma 4.3, applying a slight extension of [26] Proposition 2.1 yields, for a constant $c$ only depending on the norm of $D \mathcal{F}_{0}(U)^{-1}$,

$$
\left\|U-U^{*}\right\|_{\mathcal{Z}(\Omega)} \leq c\left\|\mathcal{F}_{0}\left(U^{*}\right)\right\|_{\mathcal{Z}(\Omega)} \leq c^{\prime}\left\|\left(\alpha\left(p^{*}\right)-\alpha^{*}\left(\cdot, p^{*}\right)\right) \boldsymbol{u}^{*}\right\|_{L^{2}(\Omega)^{d}}
$$

To conclude, we use a triangle inequality and the Lipschitz property of $\alpha$, together with the same Sobolev imbeddings as in Section 3:

$$
\begin{aligned}
\left\|\left(\alpha\left(p^{*}\right)-\alpha^{*}\left(\cdot, p^{*}\right)\right) \boldsymbol{u}^{*}\right\|_{L^{2}(\Omega)^{d}} \leq & \left\|\left(\alpha\left(p_{N}\right)-\alpha^{*}\left(\cdot, p_{N}\right)\right) \boldsymbol{u}_{N}\right\|_{L^{2}(\Omega)^{d}} \\
& +c\left(\left\|p^{*}-p_{N}\right\|_{H^{1}(\Omega)}\left\|\boldsymbol{u}^{*}\right\|_{H^{s}(\Omega)^{d}}+\left\|\boldsymbol{u}^{*}-\boldsymbol{u}_{N}\right\|_{L^{2}(\Omega)^{d}}\right) .
\end{aligned}
$$

All this gives the desired estimate.

Remark 4.5. As standard in the multistep approach, the estimate on the simplication error involves terms depending on the discretization error in its right-hand side. It seems impossible to avoid that, because the error indicator $\eta_{N, k}^{(s)}$ must be computed explicitly, hence only depends on the discrete solution.

On the other hand, the residual equation can be written explicitly by subtracting (2.11) from (2.4). It reads

$$
\begin{aligned}
\forall \boldsymbol{v} \in L^{2}(\Omega)^{d}, \quad a^{[p]}\left(\boldsymbol{u}-\boldsymbol{u}^{*}, \boldsymbol{v}\right)+b(\boldsymbol{v}, p & \left.-p^{*}\right) \\
& =-\int_{\Omega}\left(\alpha(p)-\alpha^{*}\left(\boldsymbol{x}, p^{*}\right)\right) \boldsymbol{u}^{*}(\boldsymbol{x}) \cdot \boldsymbol{v}(\boldsymbol{x}) \mathrm{d} \boldsymbol{x},
\end{aligned}
$$

$$
\forall q \in H_{(p)}^{1}(\Omega), \quad b\left(\boldsymbol{u}-\boldsymbol{u}^{*}, q\right)=0 .
$$

Thus, proving the next estimate is nearly obvious.

Proposition 4.6. If Assumption 4.2 holds, the following estimate holds for each indicator $\eta_{N, k}^{(s)}$ defined in (4.2)

$$
\eta_{N, k}^{(s)} \leq c\left(\left\|\boldsymbol{u}-\boldsymbol{u}^{*}\right\|_{L^{2}\left(\Omega_{k}\right)^{d}}+\left\|p-p^{*}\right\|_{H^{1}\left(\Omega_{k}\right)}+\left\|\boldsymbol{u}^{*}-\boldsymbol{u}_{N}\right\|_{L^{2}\left(\Omega_{k}\right)^{d}}+\left\|p^{*}-p_{N}\right\|_{H^{1}\left(\Omega_{k}\right)}\right) .
$$

Proof. By using a triangle inequality similar to (4.6), we only have to bound $\left\|\left(\alpha\left(p^{*}\right)-\alpha^{*}\left(\cdot, p^{*}\right)\right) \boldsymbol{u}^{*}\right\|_{L^{2}\left(\Omega_{k}\right)^{d}}$. A further triangle inequality yields

$$
\left\|\left(\alpha\left(p^{*}\right)-\alpha^{*}\left(\cdot, p^{*}\right)\right) \boldsymbol{u}^{*}\right\|_{L^{2}\left(\Omega_{k}\right)^{d}} \leq c\left\|p-p^{*}\right\|_{H^{1}\left(\Omega_{k}\right)}\left\|\boldsymbol{u}^{*}\right\|_{H^{s}\left(\Omega_{k}\right)^{d}}+\left\|\left(\alpha(p)-\alpha^{*}\left(\cdot, p^{*}\right)\right) \boldsymbol{u}^{*}\right\|_{L^{2}\left(\Omega_{k}\right)^{d}} .
$$

Thus, the desired estimate is obtained by taking the function $\boldsymbol{v}$ in (4.7) equal to

$$
\boldsymbol{v}_{k}= \begin{cases}\left(\alpha(p)-\alpha^{*}\left(\cdot, p^{*}\right)\right) \boldsymbol{u}^{*} & \text { in } \Omega_{k}, \\ \mathbf{0} & \text { elsewhere, }\end{cases}
$$

and using the continuity of the forms $a^{[p]}(\cdot, \cdot)$ and $b(\cdot, \cdot)$.

\subsection{Error due to the discretization}

We need some further notation: For $1 \leq k \leq K$, let $\mathcal{E}_{k}^{0}$ and $\mathcal{E}_{k}^{(f)}$ be the set of edges $(d=2)$ or faces $(d=3)$ of $\Omega_{k}$ which are not contained in $\partial \Omega$ or are contained in $\bar{\Gamma}_{(f)}$, respectively. We also introduce an approximation $g_{N}$ of $g$ defined similarly as $p_{0 N}$ : Assuming that $g$ is continuous on $\bar{\Gamma}_{(f)}$, for each edge $(d=2)$ or face $(d=3)$ $\Gamma_{\ell}$ of an element $\Omega_{k}$ which is contained in $\Gamma_{(f)},\left.g_{N}\right|_{\Gamma_{\ell}}$ belongs to $\mathbb{P}_{N}\left(\Gamma_{\ell}\right)$ and is equal to $g$ at the $(N+1)^{d-1}$ nodes $F_{k}\left(\xi_{i}, \xi_{j}\right)$ or $F_{k}\left(\xi_{i}, \xi_{j}, \xi_{m}\right)$ which are located on $\bar{\Gamma}_{\ell}$.

Next, for each $k, 1 \leq k \leq K$, we define the error indicator

$$
\begin{aligned}
\eta_{N, k}^{(d)}=\left\|\mathcal{I}_{N} \boldsymbol{f}-\alpha^{*}\left(\cdot, p_{N}\right) \boldsymbol{u}_{N}-\operatorname{grad} p_{N}\right\|_{L^{2}\left(\Omega_{k}\right)^{d}}+N^{-1}\left\|\operatorname{div} \boldsymbol{u}_{N}\right\|_{L^{2}\left(\Omega_{k}\right)} \\
\quad+\sum_{\gamma \in \mathcal{E}_{k}^{0}} N^{-\frac{1}{2}}\left\|\left[\boldsymbol{u}_{N} \cdot \boldsymbol{n}\right]_{\gamma}\right\|_{L^{2}(\gamma)}+\sum_{\gamma \in \mathcal{E}_{k}^{(f)}} N^{-\frac{1}{2}}\left\|g_{N}-\boldsymbol{u}_{N} \cdot \boldsymbol{n}\right\|_{L^{2}(\gamma)} .
\end{aligned}
$$


Indeed, all solutions $U^{*}$ of problem (2.10) - (2.11) and $U_{N}$ of problem (3.5) - (3.6) satisfy the following residual equations, for all $\boldsymbol{v}$ in $L^{2}(\Omega)^{d}$,

$$
\begin{aligned}
& a^{*\left[p^{*}\right]}\left(\boldsymbol{u}^{*}, \boldsymbol{v}\right)-a^{*\left[p_{N}\right]}\left(\boldsymbol{u}_{N}, \boldsymbol{v}\right)+b\left(\boldsymbol{v}, p^{*}-p_{N}\right) \\
& =\int_{\Omega}\left(\mathcal{I}_{N} \boldsymbol{f}-\alpha^{*}\left(\boldsymbol{x}, p_{N}\right) \boldsymbol{u}_{N}-\operatorname{grad} p_{N}\right)(\boldsymbol{x}) \cdot \boldsymbol{v}(\boldsymbol{x}) \mathrm{d} \boldsymbol{x}+\int_{\Omega}\left(\boldsymbol{f}-\mathcal{I}_{N} \boldsymbol{f}\right)(\boldsymbol{x}) \cdot \boldsymbol{v}(\boldsymbol{x}) \mathrm{d} \boldsymbol{x},
\end{aligned}
$$

and, for all $q$ in $H_{(p)}^{1}(\Omega)$,

$$
b\left(\boldsymbol{u}^{*}-\boldsymbol{u}_{N}, q\right)=\langle g, q\rangle^{(f)}-b\left(\boldsymbol{u}_{N}, q\right) .
$$

To handle this last equation, we use the second line of (3.6), together with the definition of $g_{N}$, and observe by integrating by parts on each $\Omega_{k}$ that, for any $q_{N}$ in $\mathbb{M}_{N-1}$ (with obvious notation)

$$
\begin{aligned}
b\left(\boldsymbol{u}^{*}-\right. & \left.\boldsymbol{u}_{N}, q\right)=\left\langle g-g_{N}, q\right\rangle^{(f)}+\left\langle g_{N}, q-q_{N}\right\rangle^{(f)} \\
& +\sum_{k=1}^{K}\left(\int_{\Omega_{k}}\left(\operatorname{div} \boldsymbol{u}_{N}\right)(\boldsymbol{x})\left(q-q_{N}\right)(\boldsymbol{x}) \mathrm{d} \boldsymbol{x}-\int_{\partial \Omega_{k}}\left(\boldsymbol{u}_{N} \cdot \boldsymbol{n}\right)(\boldsymbol{\tau})\left(q-q_{N}\right)(\boldsymbol{\tau}) \mathrm{d} \boldsymbol{\tau}\right) .
\end{aligned}
$$

We now make an assumption on the solution of problem (2.10)-(2.11) which is very similar but weaker than Assumption 3.1 (and requires the same notation).

Assumption 4.7. The solution $U^{*}=\left(\boldsymbol{u}^{*}, p^{*}\right)$ of problem $(2.10)-(2.11)$

(i) belongs to $H^{s}(\Omega)^{d} \times H^{s+1}(\Omega)$ for some $s>0$ in dimension $d=2$ and $s>\frac{1}{2}$ in dimension $d=3$;

(ii) is such that $D \mathcal{F}\left(U^{*}\right)$ is an isomorphism of $L^{2}(\Omega)^{d} \times H^{1}(\Omega)$.

The arguments for proving the next lemma are exactly the same as for Lemma 4.3.

Lemma 4.8. If Assumption 4.7 holds, the mapping: $V \mapsto D \mathcal{F}(V)$ is continuous on $H^{s}(\Omega)^{d} \times H^{s+1}(\Omega)$ and Lipschitz-continuous on a neighbourhood of $U$ in $\prod_{k=1}^{K} H^{s}\left(\Omega_{k}\right)^{d} \times \prod_{k=1}^{K} H^{s+1}\left(\Omega_{k}\right)$.

We also recall from [6], Lemmas 3.3 and 3.4 the next results.

Lemma 4.9. For all $q$ in $H^{1}(\Omega)$, there exists a $q_{N}$ in $\mathbb{M}_{N}$ satisfying for $1 \leq k \leq K$ and for all edges $(d=2)$ or faces $(d=3) \gamma$ of $\Omega_{k}$,

$$
\left\|q-q_{N}\right\|_{L^{2}\left(\Omega_{k}\right)} \leq c \rho(\Omega) N^{-1}\|q\|_{H^{1}(\Omega)}, \quad\left\|q-q_{N}\right\|_{L^{2}(\gamma)} \leq c N^{-\frac{1}{2}}\|q\|_{H^{1}(\Omega)},
$$

with $\rho(\Omega)$ equal to 1 if the domain $\Omega$ is either two-dimensional or convex, to $N^{\frac{1}{2}}$ otherwise.

Proposition 4.10. If Assumption 4.7 holds, there exists a neighbourhood of $U^{*}$ such that the following a posteriori error estimate holds for any solution $U_{N}=\left(\boldsymbol{u}_{N}, p_{N}\right)$ of problem (3.5)-(3.6) in this neighbourhood

$$
\begin{aligned}
\left\|\boldsymbol{u}^{*}-\boldsymbol{u}_{N}\right\|_{L^{2}(\Omega)^{d}}+ & \left\|p^{*}-p_{N}\right\|_{H^{1}(\Omega)} \leq c\left(\boldsymbol{u}^{*}, p^{*}\right)\left(\rho(\Omega)\left(\sum_{k=1}^{K}\left(\eta_{N, k}^{(d)}\right)^{2}\right)^{\frac{1}{2}}\right. \\
& \left.+\left\|\boldsymbol{f}-\mathcal{I}_{N} \boldsymbol{f}\right\|_{L^{2}(\Omega)^{d}}+\left\|p_{0}-p_{0 N}\right\|_{H^{\frac{1}{2}\left(\Gamma_{(p)}\right)}}+\left\|g-g_{N}\right\|_{H_{00}^{\frac{1}{2}\left(\Gamma_{(f)}\right)^{\prime}}}\right),
\end{aligned}
$$

where the constant $c\left(\boldsymbol{u}^{*}, p^{*}\right)$ only depends on the solution $U^{*}$.

Proof. There also, owing to Lemma 4.8, applying [26], Proposition 2.1 yields, for a constant $c$ only depending on the norm of $D \mathcal{F}\left(U^{*}\right)^{-1}$,

$$
\left\|U^{*}-U_{N}\right\|_{\mathcal{Z}(\Omega)} \leq c\left\|\mathcal{F}\left(U_{N}\right)\right\|_{\mathcal{Z}(\Omega)} \leq c\left\|\mathcal{F}\left(U^{*}\right)-\mathcal{F}\left(U_{N}\right)\right\|_{\mathcal{Z}(\Omega)} .
$$

The right-hand side is then evaluated from (4.10) and (4.12), combined with Lemma 4.9.

The converse estimate (i.e. the upper bound of each $\eta_{N, k}^{(d)}$ as a function of the error) would likely be not optimal (see [5], Thm. 2.9). We do not present it because we do not intend to perform adaptivity with respect to $N$. 


\subsection{Summary of the results}

Up to the terms involving the data, namely

$$
\left\|\boldsymbol{f}-\mathcal{I}_{N} \boldsymbol{f}\right\|_{L^{2}(\Omega)^{d}}+\left\|p_{0}-p_{0 N}\right\|_{H^{\frac{1}{2}\left(\Gamma_{(p)}\right)}}+\left\|g-g_{N}\right\|_{H_{00}^{\frac{1}{2}\left(\Gamma_{(f)}\right)}},
$$

the full error

$$
E=\left\|\boldsymbol{u}-\boldsymbol{u}^{*}\right\|_{L^{2}(\Omega)^{d}}+\left\|p-p^{*}\right\|_{H^{1}(\Omega)}+\left\|\boldsymbol{u}^{*}-\boldsymbol{u}_{N}\right\|_{L^{2}(\Omega)^{d}}+\left\|p^{*}-p_{N}\right\|_{H^{1}(\Omega)},
$$

satisfies

$$
E \leq c\left(\sum_{k=1}^{K}\left(\left(\eta_{N, k}^{(s)}\right)^{2}+\rho(\Omega)^{2}\left(\eta_{N, k}^{(d)}\right)^{2}\right)\right)^{\frac{1}{2}} .
$$

This estimate is fully optimal when the domain $\Omega$ is two-dimensional or convex. Moreover, for three-dimensional non-convex domains $\Omega$, the lack of optimality only concerns the terms $\left\|\operatorname{div} \boldsymbol{u}_{N}\right\|_{L^{2}\left(\Omega_{k}\right)}$. On the other hand, estimate (4.8) is local and proves the optimality of the indicators $\eta_{N, k}^{(s)}$. So they form an efficient tool for the automatic simplification of the model, as described in the following strategy.

A little drawback is that these estimates require Assumptions 4.2 and 4.7. However,

- part (i) of these assumptions is always satisfied in dimension $d=2$ and is likely for convex domains in dimension $d=3$;

- part (ii) of these assumptions only means that the solutions $U$ and $U^{*}$ are nonsingular in the sense of [14], which is much weaker than the uniqueness assumption.

\subsection{The adaptivity strategy}

Let $\eta^{*}$ be a fixed tolerance. From now on, we work with $N$ sufficiently large for the quantities in (4.15) to be smaller than $\eta^{*}$.

INITIALIZATION STEP. We first work with the partition of $\Omega$ given by

$$
\Omega_{\sharp}^{0}=\emptyset, \quad \Omega_{b}^{0}=\Omega,
$$

and we solve the corresponding linear problem (3.5)-(3.6).

Adaptation Step. We now assume that a partition of $\Omega$ into $\Omega_{\sharp}^{m}$ and $\Omega_{b}^{m}$ is given. We compute the corresponding solution $\left(\boldsymbol{u}_{N}, p_{N}\right)$ of problem (3.5)-(3.6), the indicators $\eta_{N, k}^{(s)}$ and their mean value $\bar{\eta}_{N}^{(s)}$, the indicators $\eta_{N, k}^{(d)}$ and their mean value $\bar{\eta}_{N}^{(d)}$. We recall that $\eta_{N, k}^{(s)}$ is not zero only if $\Omega_{k}$ is contained in $\Omega_{\mathrm{b}}^{m}$. The new partition of $\Omega$ is thus constructed in the following way:

(i) The domain $\Omega_{\sharp}^{m+1}$ is the union of $\Omega_{\sharp}^{m}$ and of all $\Omega_{k}$ such that

$$
\eta_{N, k}^{(s)} \geq \max \left\{\bar{\eta}_{N}^{(s)}, \bar{\eta}_{N}^{(d)}\right\}
$$

(ii) The domain $\Omega_{\mathrm{b}}^{m+1}$ is taken equal to $\Omega \backslash \bar{\Omega}_{\sharp}^{m+1}$.

Remark 4.11. The adaptation step can be improved in the two following ways:

(i) At each step $m$, the partition into $\Omega_{\sharp}^{m}$ and $\Omega_{b}^{m}$ can be regularized, in order to diminish the number of connected components of $\Omega_{\sharp}^{m}$ and $\Omega_{b}^{m}$. For instance, if a domain $\Omega_{k}$ in $\Omega_{b}^{m}$ is surrounded by domains in $\Omega_{\sharp}^{m}$, it can be inserted into $\Omega_{\sharp}^{m}$.

(ii) When a domain $\Omega_{k}$ of very large size must be inserted into $\Omega_{\sharp}^{m}$, an idea is to consider a new decomposition of $\Omega$ into subdomains where this $\Omega_{k}$ is replaced by smaller $\Omega_{k^{\prime}}$ and to perform a new computation in order to determine which of these subdomains must be inserted into $\Omega_{\sharp}^{m}$.

The adaptation step must be iterated either a fixed number of times or until the Hilbertian sum $\left(\sum_{k=1}^{K}\left(\eta_{N, k}^{(s)}\right)^{2}\right)^{\frac{1}{2}}$ becomes smaller than $\eta^{*}$ (when possible). 


\section{An iterative Algorithm}

A large number of iterative algorithms exists for solving nonlinear problems of the type considered here, for instance the Newton's method (we refer to [14] for the proof of its convergence which relies on the arguments in Sect. 3, see also [20], Chap. IV, Thm. 6.3). However most of them require to compute the derivative $\partial_{\xi} \alpha$ which is not always possible. So, we now present a low cost algorithm and investigate its convergence.

Assuming that an initial guess $\left(\boldsymbol{u}_{N}^{0}, p_{N}^{0}\right)$ is given (for instance, it can be the solution of problem (3.5)-(3.6) with $\alpha^{*}\left(\cdot, p_{N}\right)$ replaced by $\left.\alpha_{0}\right)$, we solve iteratively the problems

Find $\left(\boldsymbol{u}_{N}^{n}, p_{N}^{n}\right)$ in $\mathbb{X}_{N} \times \mathbb{M}_{N}$ such that

$$
p_{N}^{n}=p_{0 N} \quad \text { on } \Gamma_{(p)},
$$

and

$$
\begin{aligned}
& \forall \boldsymbol{v}_{N} \in \mathbb{X}_{N}, \quad a_{N}^{*\left[p_{N}^{n-1}\right]}\left(\boldsymbol{u}_{N}^{n}, \boldsymbol{v}_{N}\right)+b_{N}\left(\boldsymbol{v}_{N}, p_{N}^{n}\right)=\left(\left(\boldsymbol{f}, \boldsymbol{v}_{N}\right)\right)_{N}, \\
& \forall q_{N} \in \mathbb{M}_{N}^{(p)}, \quad b_{N}\left(\boldsymbol{u}_{N}^{n}, q_{N}\right)=\left(\left(g, q_{N}\right)\right)_{N}^{(f)} .
\end{aligned}
$$

It is readily checked that this problem admits a unique solution. Proving the convergence of the sequence $\left(\boldsymbol{u}_{N}^{n}, p_{N}^{n}\right)_{n}$ requires some preliminary lemmas.

Lemma 5.1. When all assumptions of Theorem 3.7 hold, there exists a constant $\lambda$ only depending on $U^{*}$ such that any solution $\left(\boldsymbol{u}_{N}, p_{N}\right)$ of problem (3.5)-(3.6) satisfies

$$
\left\|\boldsymbol{u}_{N}\right\|_{L^{\rho}(\Omega)^{d}} \leq \lambda,
$$

with $\rho>2$ in dimension $d=2$ and $\rho=3$ in dimension $d=3$.

Proof. We again use the approximation $\left(\boldsymbol{v}_{N}^{*}, q_{N}^{*}\right)$ of $\left(\boldsymbol{u}^{*}, p^{*}\right)$ which satisfies (3.21). Indeed, we have

$$
\left\|\boldsymbol{u}_{N}\right\|_{L^{\rho}(\Omega)^{d}} \leq\left\|\boldsymbol{v}_{N}^{*}\right\|_{L^{\rho}(\Omega)^{d}}+\left\|\boldsymbol{u}_{N}-\boldsymbol{v}_{N}^{*}\right\|_{L^{\rho}(\Omega)^{d}} .
$$

Evaluating the first term results from (3.21) and the imbedding of $H^{s}(\Omega)$ into $L^{\rho}(\Omega)$. To bound the second one, we use an inverse inequality, see [9], Chapter III, Proposition 2.1. All this yields

$$
\left\|\boldsymbol{u}_{N}\right\|_{L^{\rho}(\Omega)^{d}} \leq\left\|\boldsymbol{u}^{*}\right\|_{H^{s}(\Omega)^{d}}+c N^{2 d\left(\frac{1}{2}-\frac{1}{\rho}\right)}\left(\left\|\boldsymbol{u}^{*}-\boldsymbol{v}_{N}^{*}\right\|_{L^{2}(\Omega)^{d}}+\left\|\boldsymbol{u}^{*}-\boldsymbol{u}_{N}\right\|_{L^{2}(\Omega)^{d}}\right) .
$$

The quantity $\left\|\boldsymbol{u}^{*}-\boldsymbol{v}_{N}^{*}\right\|_{L^{2}(\Omega)^{d}}$ is bounded in (3.21), while the estimate for $\left\|\boldsymbol{u}^{*}-\boldsymbol{u}_{N}\right\|_{L^{2}(\Omega)^{d}}$ is stated in Theorem 3.7 (only this requires Assumption 3.1). To conclude, we choose $\rho$ in dimension $d=2$ such that $2 d\left(\frac{1}{2}-\frac{1}{\rho}\right)-s=0$.

When subtracting equation (5.2) at step $n$ from equation (3.6) we obtain

$$
\begin{aligned}
\forall \boldsymbol{v}_{N} \in \mathbb{X}_{N}, \quad a_{N}^{*\left[p_{N}^{n-1}\right]}\left(\boldsymbol{u}_{N}-\boldsymbol{u}_{N}^{n}, \boldsymbol{v}_{N}\right)+b_{N}\left(\boldsymbol{v}_{N}, p_{N}-p_{N}^{n}\right) & =\left(\left(\left(\alpha^{*}\left(\cdot, p_{N}^{n-1}\right)-\alpha^{*}\left(\cdot, p_{N}\right)\right) \boldsymbol{u}_{N}, \boldsymbol{v}_{N}\right)\right)_{N}, \\
\forall q_{N} \in \mathbb{M}_{N}^{(p)}, \quad b_{N}\left(\boldsymbol{u}_{N}-\boldsymbol{u}_{N}^{n}, q_{N}\right)=0 . &
\end{aligned}
$$

We are thus in a position to derive the next result.

Lemma 5.2. When all assumptions of Theorem 3.7 hold, the sequence $\left(\boldsymbol{u}_{N}^{n}, p_{N}^{n}\right)_{n}$ satisfies the following estimate

$$
\begin{aligned}
\left\|\boldsymbol{u}_{N}-\boldsymbol{u}_{N}^{n}\right\|_{L^{2}(\Omega)^{d}} \leq & c \frac{\lambda \alpha^{\dagger}}{\alpha_{1}}\left\|p_{N}-p_{N}^{n-1}\right\|_{H^{1}\left(\Omega_{\sharp}\right)}, \\
& \left\|p_{N}-p_{N}^{n}\right\|_{H^{1}(\Omega)} \leq c \lambda \alpha^{\dagger}\left(1+\frac{\alpha_{2}}{\alpha_{1}}\right)\left\|p_{N}-p_{N}^{n-1}\right\|_{H^{1}\left(\Omega_{\sharp}\right)},
\end{aligned}
$$

where $\alpha^{\dagger}$ stands for the Lipschitz constant of the function $\alpha$. 
Proof. We take $\boldsymbol{v}_{N}$ equal to $\boldsymbol{u}_{N}-\boldsymbol{u}_{N}^{n}$ in (5.4). The standard properties of the discrete product $((\cdot, \cdot))_{N}($ see [10], Rem. 13.3 for instance), combined with a Cauchy-Schwarz inequality (note also that $p_{N}-p_{N}^{n}$ belongs to $\left.\mathbb{M}_{N}^{(p)}\right)$, thus yield

$$
\alpha_{1}\left\|\boldsymbol{u}_{N}-\boldsymbol{u}_{N}^{n}\right\|_{L^{2}(\Omega)^{d}} \leq\left(\left(\left(\alpha^{*}\left(\cdot, p_{N}^{n-1}\right)-\alpha^{*}\left(\cdot, p_{N}\right)\right) \boldsymbol{u}_{N},\left(\alpha^{*}\left(\cdot, p_{N}^{n-1}\right)-\alpha^{*}\left(\cdot, p_{N}\right)\right) \boldsymbol{u}_{N}\right)\right)_{N}^{\frac{1}{2}} .
$$

It follows from the definition of the discrete scalar product together with the Lipschitz property of $\alpha$ that

$$
\alpha_{1}\left\|\boldsymbol{u}_{N}-\boldsymbol{u}_{N}^{n}\right\|_{L^{2}(\Omega)^{d}} \leq \alpha^{\dagger}\left(\left(\left(p_{N}^{n-1}-p_{N}\right) \boldsymbol{u}_{N},\left(p_{N}^{n-1}-p_{N}\right) \boldsymbol{u}_{N}\right)\right)_{N}^{\frac{1}{2}} .
$$

Note that, in this product, each $\left(p_{N}^{n-1}-p_{N}\right) \boldsymbol{u}_{N}$ can be replaced by its interpolate in $\mathbb{X}_{N}$. Using once more the stability of the operator $\mathcal{I}_{N}$ on polynomials of degree $\leq 2 N$ (see [10], Rem. 13.5), we obtain, for the $\rho$ introduced in Lemma 5.1 and with $\frac{1}{\rho}+\frac{1}{\rho^{\prime}}=\frac{1}{2}$,

$$
\alpha_{1}\left\|\boldsymbol{u}_{N}-\boldsymbol{u}_{N}^{n}\right\|_{L^{2}(\Omega)^{d}} \leq c \alpha^{\dagger}\left\|p_{N}^{n-1}-p_{N}\right\|_{L^{\rho^{\prime}}(\Omega)}\left\|\boldsymbol{u}_{N}\right\|_{L^{\rho}(\Omega)^{d}} .
$$

Lemma 5.1 and the imbedding of $H^{1}(\Omega)$ into $L^{\rho^{\prime}}(\Omega)$ then yield

$$
\alpha_{1}\left\|\boldsymbol{u}_{N}-\boldsymbol{u}_{N}^{n}\right\|_{L^{2}(\Omega)^{d}} \leq c \lambda \alpha^{\dagger}\left\|p_{N}-p_{N}^{n-1}\right\|_{H^{1}(\Omega)} .
$$

All this leads to the first estimate in (5.5). To prove the second one, we need a more precise form of the inf-sup condition (3.18): Taking $\boldsymbol{v}_{N}$ equal to $\operatorname{grad}\left(p_{N}-p_{N}^{n}\right)$ in (5.4) gives, thanks to the same arguments as previously and a Poincaré-Friedrichs inequality,

$$
\begin{aligned}
\left\|p_{N}-p_{N}^{n}\right\|_{H^{1}(\Omega)} \leq & c\left\|\boldsymbol{u}_{N}-\boldsymbol{u}_{N}^{n}\right\|_{L^{2}(\Omega)^{d}} \\
& +c^{\prime}\left(\left(\left(\alpha^{*}\left(\cdot, p_{N}^{n-1}\right)-\alpha^{*}\left(\cdot, p_{N}\right)\right) \boldsymbol{u}_{N},\left(\alpha^{*}\left(\cdot, p_{N}^{n-1}\right)-\alpha^{*}\left(\cdot, p_{N}\right)\right) \boldsymbol{u}_{N}\right)\right)_{N}^{\frac{1}{2}} .
\end{aligned}
$$

The two terms in the right-hand side have been evaluated above, which leads to the second estimate.

The geometric convergence of the method can now be easily derived with a further assumption.

Proposition 5.3. When all assumptions of Theorem 3.7 hold, there exists a positive constant $c_{0}$ independent of $N$ such that, if

$$
\lambda \alpha^{\dagger}\left(1+\frac{\alpha_{2}}{\alpha_{1}}\right)<c_{0}
$$

the sequence $\left(\boldsymbol{u}_{N}^{n}, p_{N}^{n}\right)_{n}$ converges to $\left(\boldsymbol{u}_{N}, p_{N}\right)$ in $L^{2}(\Omega)^{d} \times H^{1}(\Omega)$. Moreover, the following estimate holds with the constant $\kappa$ equal to $\lambda \alpha^{\dagger}\left(1+\frac{\alpha_{2}}{\alpha_{1}}\right) c_{0}^{-1}$,

$$
\begin{aligned}
\left\|\boldsymbol{u}_{N}-\boldsymbol{u}_{N}^{n}\right\|_{L^{2}(\Omega)^{d}} \leq & c \frac{\lambda \alpha^{\dagger}}{\alpha_{1}} \kappa^{n-1}\left\|p_{N}-p_{N}^{0}\right\|_{H^{1}\left(\Omega_{\sharp}\right)}, \\
& \left\|p_{N}-p_{N}^{n}\right\|_{H^{1}(\Omega)} \leq \kappa^{n}\left\|p_{N}-p_{N}^{0}\right\|_{H^{1}\left(\Omega_{\sharp}\right)},
\end{aligned}
$$

where $\alpha^{\dagger}$ stands for the Lipschitz constant of the function $\alpha$.

Remark 5.4. It can be noted [3], Proposition 2.4 that Assumption (5.6) is exactly the sufficient condition for problems $(2.10)-(2.11)$ and also $(3.5)-(3.6)$ to have a unique solution. So it is logical that no further assumption is enforced on the initial guess $p_{N}^{0}$ to obtain the convergence. 
Assumption (5.6) mainly means that the function $\alpha$ does not present high variations, i.e., that the coefficient $\alpha^{\dagger}$ is small enough.

We now perform the a posteriori analysis for the iterative algorithm. We follow the approach proposed in [19], even if our arguments are different. We recall that any solution of (3.5)-(3.6) satisfies (3.14). Similarly, it is readily checked that the solution $U_{N}^{n}=\left(\boldsymbol{u}_{N}^{n}, p_{N}^{n}\right)$ of problem (5.1)-(5.2) satisfies

$$
\mathcal{F}_{N}\left(U_{N}^{n}\right)=U_{N}^{n}-\mathcal{T}_{N} \mathcal{G}_{N}\left(U_{N}^{n}\right)=\mathcal{T}_{N}\left(\boldsymbol{R}_{N}^{n}, 0,0\right),
$$

the residual $\boldsymbol{R}_{N}^{n}$ being given by

$$
\forall \boldsymbol{v}_{N} \in \mathbb{X}_{N}, \quad \int_{\Omega} \boldsymbol{R}_{N}^{n}(\boldsymbol{x}) \cdot \boldsymbol{v}_{N}(\boldsymbol{x}) \mathrm{d} \boldsymbol{x}=\left(\left(\left(\alpha^{*}\left(\cdot, p_{N}^{n-1}\right)-\alpha^{*}\left(\cdot, p_{N}^{n}\right)\right) \boldsymbol{u}_{N}^{n}, \boldsymbol{v}_{N}\right)\right)_{N} .
$$

In view of the previous equations, in each domain $\Omega_{k}, 1 \leq k \leq K$, we define the error indicator

$$
\eta_{N, k, n}^{(i a)}=\left\|\mathcal{I}_{N}\left(\alpha^{*}\left(\cdot, p_{N}^{n}\right)-\alpha^{*}\left(\cdot, p_{N}^{n-1}\right)\right) \boldsymbol{u}_{N}^{n}\right\|_{L^{2}\left(\Omega_{k}\right)^{d}} .
$$

Here, all $\eta_{N, k, n}^{(i a)}$ such that $\Omega_{k}$ is contained in $\Omega_{b}$ are zero.

Remark 5.5. The quantity $\|\cdot\|_{N, k}$ defined by

$$
\left\|\varphi_{N}\right\|_{N, k}^{2}=\left(\varphi_{N}, \varphi_{N}\right)_{N}^{k}
$$

is obviously a norm on $\mathbb{P}_{N}\left(\Omega_{k}\right)$ (see [11], Chap. IV, Cor. 1.10). Replacing the norm $\|\cdot\|_{L^{2}\left(\Omega_{k}\right)}$ by this new norm in (5.10) makes the computation of the $\eta_{N, k, n}^{(i a)}$ easier, and all the following results still hold for this new definition.

We need some further lemmas which are very similar to Lemmas 3.4 and 3.5. The first one is a direct consequence of Lemma 3.4, see [20], Chapter IV, Theorem 3.1.

Lemma 5.6. Let $N^{*}$ denote the integer introduced in Theorem 3.7. If the coefficient $\alpha$ is of class $\mathscr{C}^{2}$ on $\mathbb{R}$ with bounded derivatives and Assumption 3.1 holds, for all $N \geq N^{*}$, the operator $D \mathcal{F}_{N}\left(U_{N}\right)$, where $U_{N}$ stands for the solution of problem (3.5)-(3.6) exhibited in Theorem 3.7, is an isomorphism of $\mathbb{X}_{N} \times \mathbb{M}_{N}$, with the norm of its inverse bounded independently of $N$.

Exactly the same arguments as for Lemma 3.5 yield the next result.

Lemma 5.7. If the coefficient $\alpha$ is of class $\mathscr{C}^{2}$ on $\mathbb{R}$ with bounded derivatives and Assumption 3.1 holds, there exist a neighbourhood of $U_{N}$ in $\mathcal{Z}_{N}$ and a constant $\lambda^{*}>0$ such that the operator $D \mathcal{F}_{N}$ satisfies the following Lipschitz property, for all $V_{N}$ in this neighbourhood,

$$
\left\|D \mathcal{F}_{N}\left(U_{N}\right)-D \mathcal{F}_{N}\left(V_{N}\right)\right\|_{\mathcal{E}} \leq \lambda^{*} \mu(N)\left\|U_{N}-V_{N}\right\|_{\mathcal{Z}(\Omega)},
$$

with $\mu(N)$ equal to $|\log N|^{\frac{1}{2}}$ in dimension $d=2$ and to $N$ in dimension $d=3$.

We are thus in a position to derive the first a posteriori error estimate.

Proposition 5.8. If the coefficient $\alpha$ is of class $\mathscr{C}^{2}$ on $\mathbb{R}$ with bounded derivatives and Assumption 3.1 holds, there exists a constant $\nu$ such that the following a posteriori error estimate holds for any solution $U_{N}^{n}=\left(\boldsymbol{u}_{N}^{n}, p_{N}^{n}\right)$ of problem (5.1)-(5.2) in the ball with centre $U_{N}$ and radius $\nu \mu(N)^{-1}$,

$$
\left\|\boldsymbol{u}_{N}-\boldsymbol{u}_{N}^{n}\right\|_{L^{2}(\Omega)^{d}}+\left\|p_{N}-p_{N}^{n}\right\|_{H^{1}(\Omega)} \leq c\left(\sum_{k=1}^{K}\left(\eta_{N, k, n}^{(i a)}\right)^{2}\right)^{\frac{1}{2}},
$$

where the constant $c$ is independent of $N$. 
Proof. Applying once more [26], Proposition 2.1 gives

$$
\left\|U_{N}-U_{N}^{n}\right\|_{\mathcal{Z}(\Omega)} \leq c\left\|\boldsymbol{R}_{N}^{n}\right\|_{L^{2}(\Omega)^{d}},
$$

where, owing to Lemma 5.6, the constant $c$ is bounded independently of $N$. Evaluting $\boldsymbol{R}_{N}^{n}$ follows from now standard arguments.

To prove the converse estimate, we observe that equations (5.8) and (5.9) can equivalently be written as

$$
\begin{array}{r}
\forall \boldsymbol{v}_{N} \in \mathbb{X}_{N}, \quad a_{N}^{\left[p_{N}\right]}\left(\boldsymbol{u}_{N}, \boldsymbol{v}_{N}\right)-a_{N}^{\left[p_{N}^{n}\right]}\left(\boldsymbol{u}_{N}^{n}, \boldsymbol{v}_{N}\right)+b_{N}\left(\boldsymbol{v}_{N}, p_{N}-p_{N}^{n}\right) \\
=\left(\left(\left(\alpha^{*}\left(\cdot, p_{N}^{n-1}\right)-\alpha^{*}\left(\cdot, p_{N}^{n}\right)\right) \boldsymbol{u}_{N}^{n}, \boldsymbol{v}_{N}\right)\right)_{N} .
\end{array}
$$

The next proposition can easily be derived from this equation.

Proposition 5.9. If the coefficient $\alpha$ is of class $\mathscr{C}^{2}$ on $\mathbb{R}$ with bounded derivatives and Assumption 3.1 holds, the following estimate holds for each indicator $\eta_{N, k, n}^{(i a)}$ defined in $(5.10)$

$$
\eta_{N, k, n}^{(i a)} \leq c\left(\left\|\boldsymbol{u}_{N}-\boldsymbol{u}_{N}^{n}\right\|_{L^{2}\left(\Omega_{k}\right)^{d}}+\left\|p_{N}-p_{N}^{n}\right\|_{H^{1}\left(\Omega_{k}\right)}\right) .
$$

Proof. We take the function $\boldsymbol{v}_{N}$ in (5.13) equal to

$$
\boldsymbol{v}_{k}= \begin{cases}\mathcal{I}_{N}\left(\left(\alpha^{*}\left(\cdot, p_{N}^{n-1}\right)-\alpha^{*}\left(\cdot, p_{N}^{n}\right)\right) \boldsymbol{u}_{N}^{n}\right) & \text { in } \Omega_{k}, \\ \mathbf{0} & \text { elsewhere. }\end{cases}
$$

Thus, we derive the estimate by using the triangle inequality

$$
a_{N}^{\left[p_{N}\right]}\left(\boldsymbol{u}_{N}, \boldsymbol{v}_{N}\right)-a_{N}^{\left[p_{N}^{n}\right]}\left(\boldsymbol{u}_{N}^{n}, \boldsymbol{v}_{N}\right)=a_{N}^{\left[p_{N}\right]}\left(\boldsymbol{u}_{N}, \boldsymbol{v}_{N}\right)-a_{N}^{\left[p_{N}^{n}\right]}\left(\boldsymbol{u}_{N}, \boldsymbol{v}_{N}\right)+a_{N}^{\left[p_{N}^{n}\right]}\left(\boldsymbol{u}_{N}-\boldsymbol{u}_{N}^{n}, \boldsymbol{v}_{N}\right),
$$

and combining the Lipschitz property of $\alpha$ with Lemma 5.1.

Estimates (5.12) and (5.14) are fully optimal and would allow us to check the convergence or non convergence of the iterative algorithm when condition (5.6) is not satisfied.

Of course, the definition of the indicators $\eta_{N, k}^{(d)}$ is now meaningless since the discrete solution $\left(\boldsymbol{u}_{N}, p_{N}\right)$ is never computed exactly. So let us introduce the modified indicators, now depending on $n$,

$$
\begin{aligned}
\eta_{N, k, n}^{(d)}=\left\|\mathcal{I}_{N} \boldsymbol{f}-\alpha^{*}\left(\cdot, p_{N}^{n-1}\right) \boldsymbol{u}_{N}^{n}-\operatorname{grad} p_{N}^{n}\right\|_{L^{2}\left(\Omega_{k}\right)^{d}}+N^{-1}\left\|\operatorname{div} \boldsymbol{u}_{N}^{n}\right\|_{L^{2}\left(\Omega_{k}\right)} \\
\quad+\sum_{\gamma \in \mathcal{E}_{k}^{0}} N^{-\frac{1}{2}}\left\|\left[\boldsymbol{u}_{N}^{n} \cdot \boldsymbol{n}\right]_{\gamma}\right\|_{L^{2}(\gamma)}+\sum_{\gamma \in \mathcal{E}_{k}^{(f)}} N^{-\frac{1}{2}}\left\|g_{N}-\boldsymbol{u}_{N}^{n} \cdot \boldsymbol{n}\right\|_{L^{2}(\gamma)} .
\end{aligned}
$$

Indeed, exactly the same arguments as for Proposition 4.10 leads to the following statement.

Proposition 5.10. If Assumption 4.7 holds, there exists a neighbourhood of $U^{*}$ such that the following a posteriori error estimate holds for any solution $U_{N}^{n}=\left(\boldsymbol{u}_{N}^{n}, p_{N}^{n}\right)$ of problem (5.1)-(5.2) in this neighbourhood

$$
\begin{aligned}
\left\|\boldsymbol{u}^{*}-\boldsymbol{u}_{N}^{n}\right\|_{L^{2}(\Omega)^{d}}+ & \left\|p^{*}-p_{N}^{n}\right\|_{H^{1}(\Omega)} \leq c\left(\boldsymbol{u}^{*}, p^{*}\right)\left(\rho(\Omega)\left(\sum_{k=1}^{K}\left(\eta_{N, k, n}^{(d)}\right)^{2}\right)^{\frac{1}{2}}\right. \\
& \left.+\left\|\boldsymbol{f}-\mathcal{I}_{N} \boldsymbol{f}\right\|_{L^{2}(\Omega)^{d}}+\left\|p_{0}-p_{0 N}\right\|_{H^{\frac{1}{2}\left(\Gamma_{(p)}\right)}}+\left\|g-g_{N}\right\|_{H_{00}^{\frac{1}{2}\left(\Gamma_{(f)}\right)^{\prime}}}\right),
\end{aligned}
$$

where the constant $c\left(\boldsymbol{u}^{*}, p^{*}\right)$ only depends on the solution $U^{*}$. 
In view of all these results, computing the $\eta_{N, k, n}^{(d)}$ at each iteration $n$ seems completely useless. Indeed, if the algorithm converges, the sequence $\left(\boldsymbol{u}_{N}^{n}, p_{N}^{n}\right)_{n}$ converges to $\left(\boldsymbol{u}_{N}, p_{N}\right)$, so that the $\eta_{N, k, n}^{(d)}$ decrease toward $\eta_{N, k}^{(d)}$. Thus, our adaptivity strategy is now very simple: For a given tolerance $\eta^{*}$ (it could be the same as in Sect. 4.4 or not),

(i) iterate the algorithm until

$$
\left(\sum_{k=1}^{K}\left(\eta_{N, k, n}^{(i a)}\right)^{2}\right)^{\frac{1}{2}} \leq \eta^{*} .
$$

(ii) if $n_{\dagger}$ denotes the smallest value of $n$ such that (5.17) holds, perform the adaptivity strategy described in Section 4.4 with each $\eta_{N, k}^{(d)}$ replaced by $\eta_{N, k, n_{\dagger}}^{(d)}$.

\section{NumERICAL EXPERIMENTS}

We check successively the efficiency of the indicators $\eta_{n, k, n}^{(i a)}$ to stop the iterative algorithm at the right iteration, next the efficiency of the adaptivity strategy proposed in Section 4.4 to construct a correct domain $\Omega_{\sharp}$. Our experiments are made in dimension $d=2$ and for rectangular domains.

\subsection{Stopping the iterative algorithm}

In a first step, to check the efficiency of the iterative algorithm, we work with $\Omega_{\sharp}=\Omega$, this $\Omega$ being the simple domain

$$
\Omega=] 0.6,3[\times]-6,0\left[, \quad \Gamma_{(p)}=\{0.6\} \times\right]-6,0\left[, \quad \Gamma_{(f)}=\partial \Omega \backslash \bar{\Gamma}_{(p)} .\right.
$$

We consider the given solution

$$
\boldsymbol{u}(x, y)=\left(\begin{array}{c}
\sin (x) \cos (y) \\
-\cos (x) \sin (y)
\end{array}\right), \quad p(x, y)=\left(\frac{y}{6}\right)^{4} .
$$

The function $\alpha$ is equal to

$$
\alpha(\xi)=\exp (\xi)
$$

truncated at $\alpha_{1}=\frac{3}{4}$ and $\alpha_{2}=3$, and the data can easily be computed from this.

The discretization is made in the following way: We cut the rectangle $\Omega$ into $K=9$ equal rectangles, three in each direction. We take the discretization parameter $N$ equal to 12 and fix the tolerance $\eta^{*}$ to $10^{-8}$.

We now denote by $\eta_{n}^{(d)}$ and $\eta_{n}^{(i a)}$ the Hilbertian sum of the indicators $\eta_{N, k, n}^{(d)}$ and $\eta_{N, k, n}^{(i a)}$, respectively, on $k$ in $\{1, \ldots, 9\}$ for $N=12$ at the iteration $n$. Figure 1 represents the sums $\eta_{n}^{(d)}$ (plain line) and $\eta_{n}^{(i a)}$ (dashed line) as a function of the iteration $n$. The convergence of the method is rather fast and both indicators decrease as a function of $n$ in a very similar way. These curves are in good coherence with the results of Section 5 .

\subsection{Convergence of the simplification}

We now work on the domain

$$
\Omega=]-1,1\left[^{2}, \quad \Gamma_{(p)}=\{-1\} \times\right]-1,1\left[, \quad \Gamma_{(f)}=\partial \Omega \backslash \bar{\Gamma}_{(p)},\right.
$$

and we consider the given solution

$$
\boldsymbol{u}(x, y)=\left(\begin{array}{c}
\sin (x) \cos (y) \\
-\cos (x) \sin (y)
\end{array}\right), \quad p(x, y)=\exp \left(-\frac{(x+1)^{2}+(y+1)^{2}}{0.05}\right) .
$$

Indeed, the fact that the pressure presents high variations only on a part of the domain (see Fig. 2) seems well appropriate for studying a possible simplification of the problem. The function $\alpha$ is still given by (6.3) and the constant $\alpha_{0}$ is taken equal to 1 . 


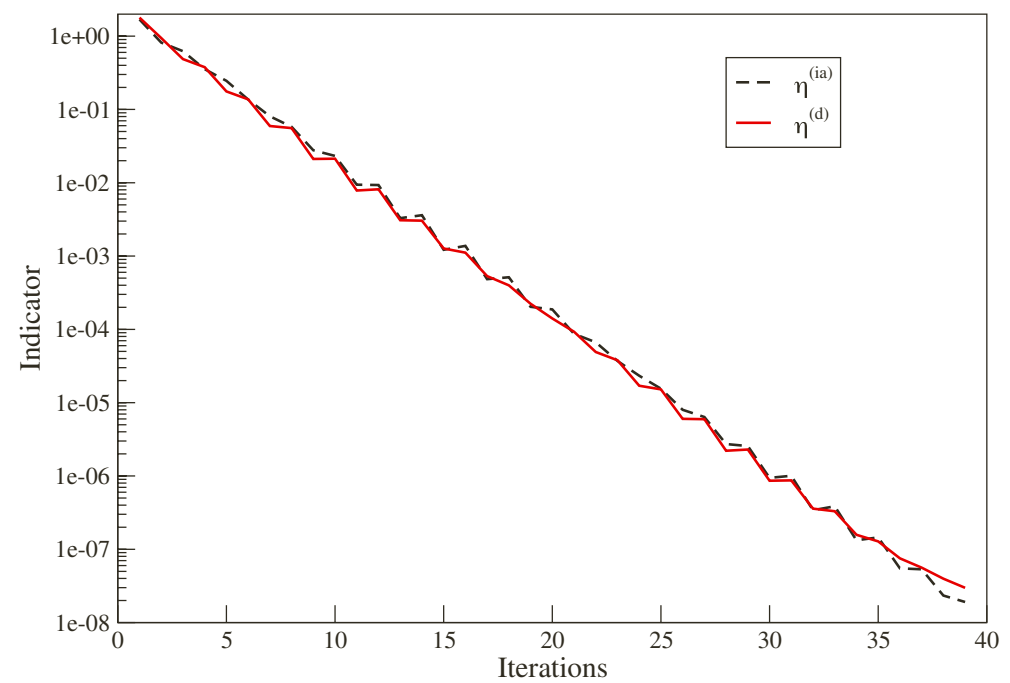

FiguRE 1. The convergence of the indicators for $\alpha$ in (6.3).
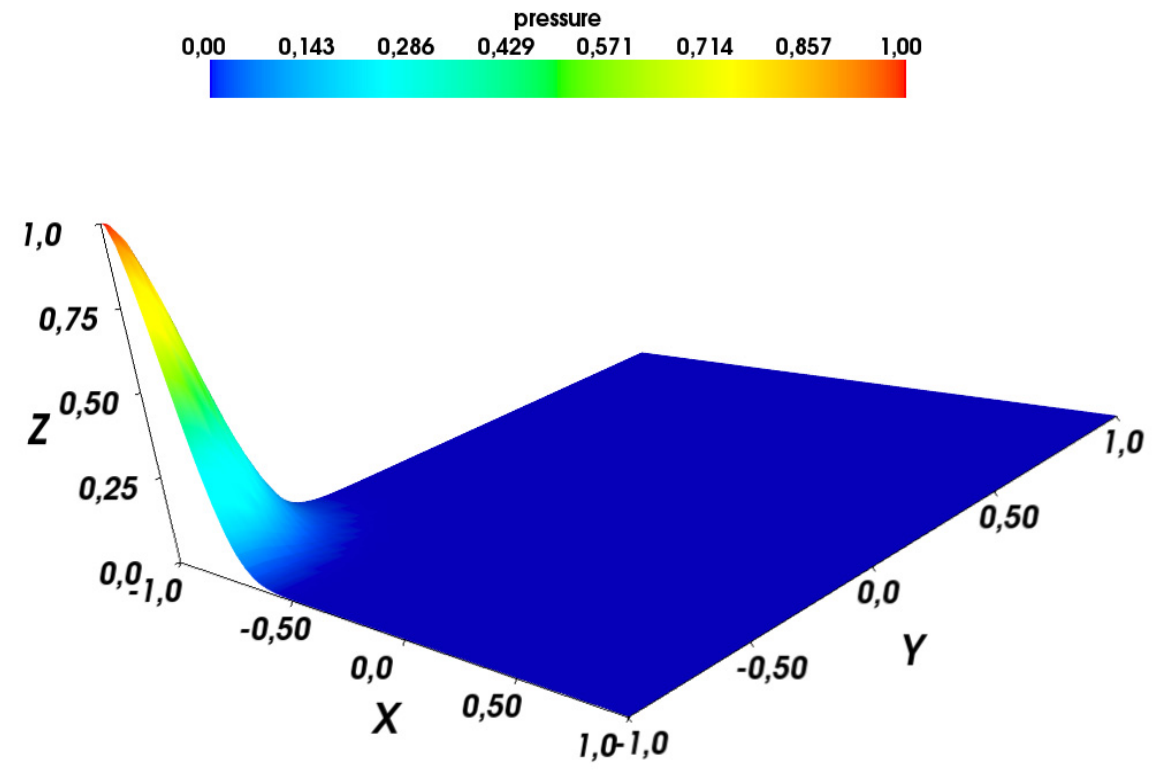

Figure 2. The pressure defined in (6.5).

The discretization here is performed with low degree polynomials: $N=4$ and much more elements: $K=$ $324=18^{2}$ equal squares. We follow the adaptivity strategy proposed in Section 4.4 , still with $\eta^{*}=10^{-8}$, and present in Figure 3 the successive partitions of $\Omega$ into $\Omega_{\sharp}^{m}$ (white) and $\Omega_{b}^{m}$ (black) for $m$ varying between 1 and 9 . The convergence is obtained for $m=9$, which proves the efficiency of our strategy. It can be noted that $\Omega_{\sharp}^{9}$ contains 22 elements.

We finally present in Figure 4 the values of the function $\alpha^{*}$ at the final iteration. 


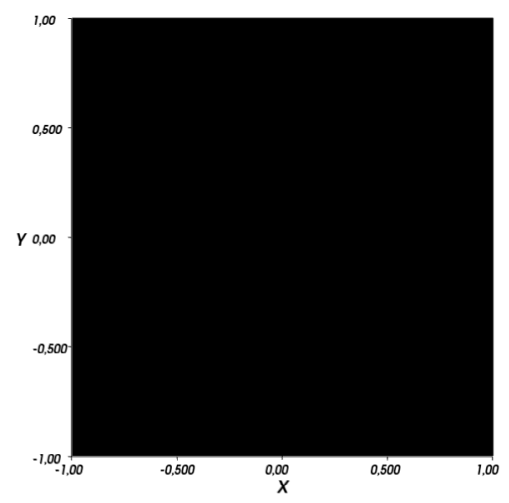

$\mathrm{m}=1$

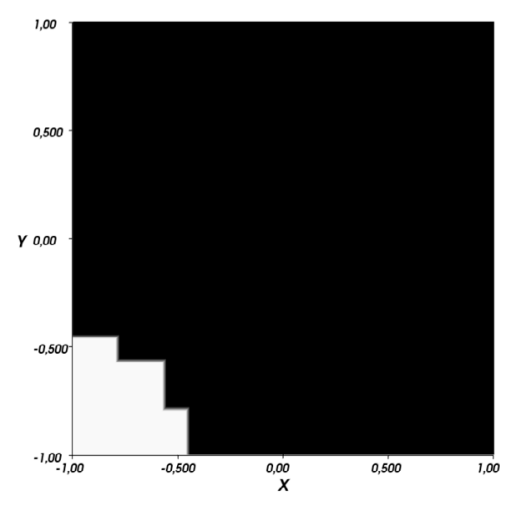

$\mathrm{m}=4$

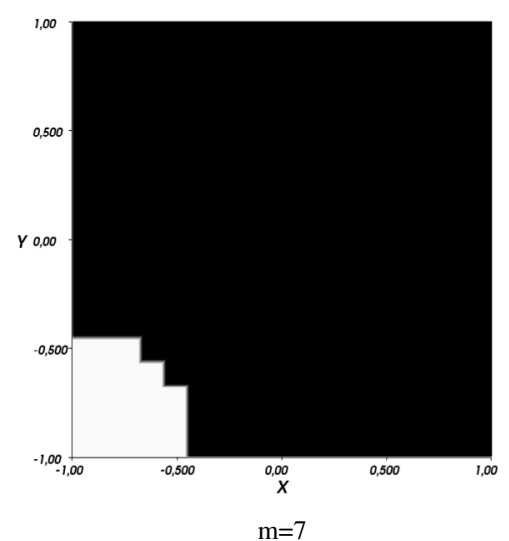

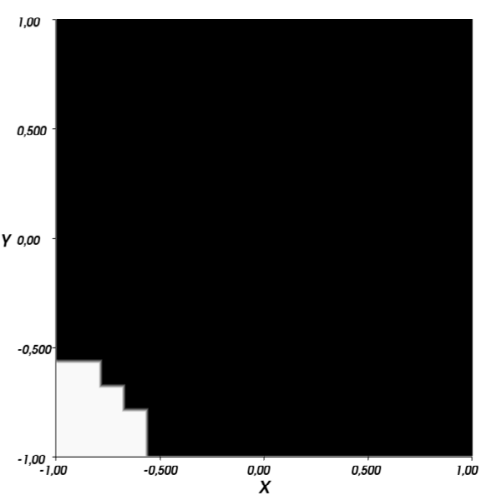

$\mathrm{m}=2$

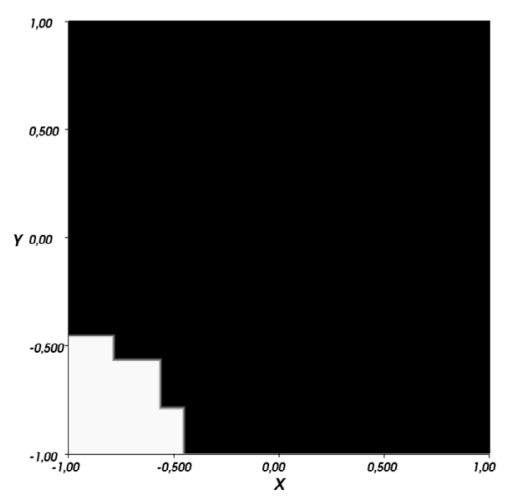

$\mathrm{m}=5$

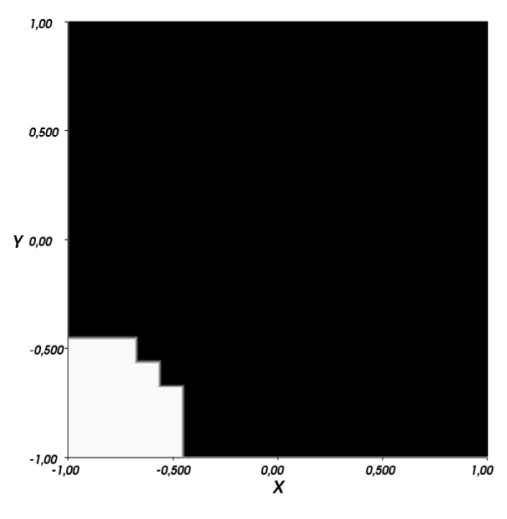

$\mathrm{m}=8$

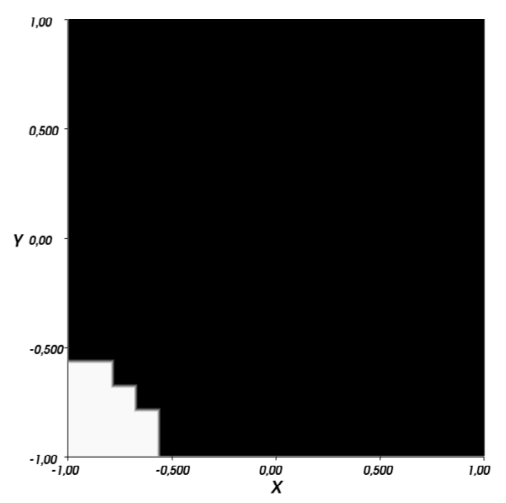

$\mathrm{m}=3$

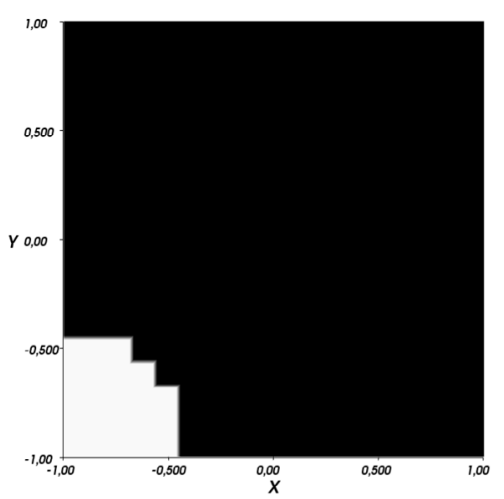

$\mathrm{m}=6$

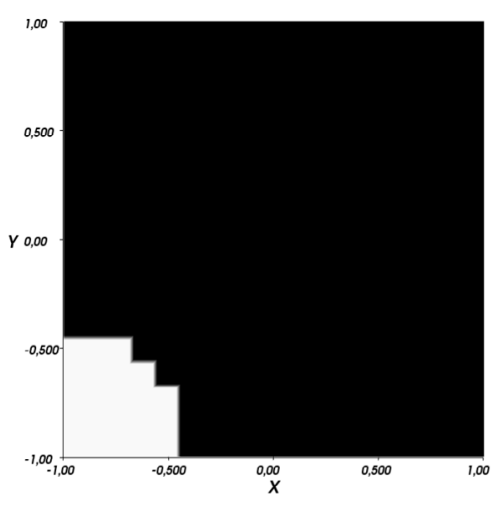

$\mathrm{m}=9$

FiguRE 3 . The successive partitions of $\Omega$ into $\Omega_{\sharp}$ and $\Omega_{\mathrm{b}}$.

\subsection{Interest of the simplification}

In order to minimize the computational time for the simplified model, we now adapt the iterative algorithm to the partition of the domain, in the following way: Each iteration is applied on $\Omega_{\sharp}$ and only one iteration over 4 is applied on the whole domain. It can be observed that this does not affect its convergence. 


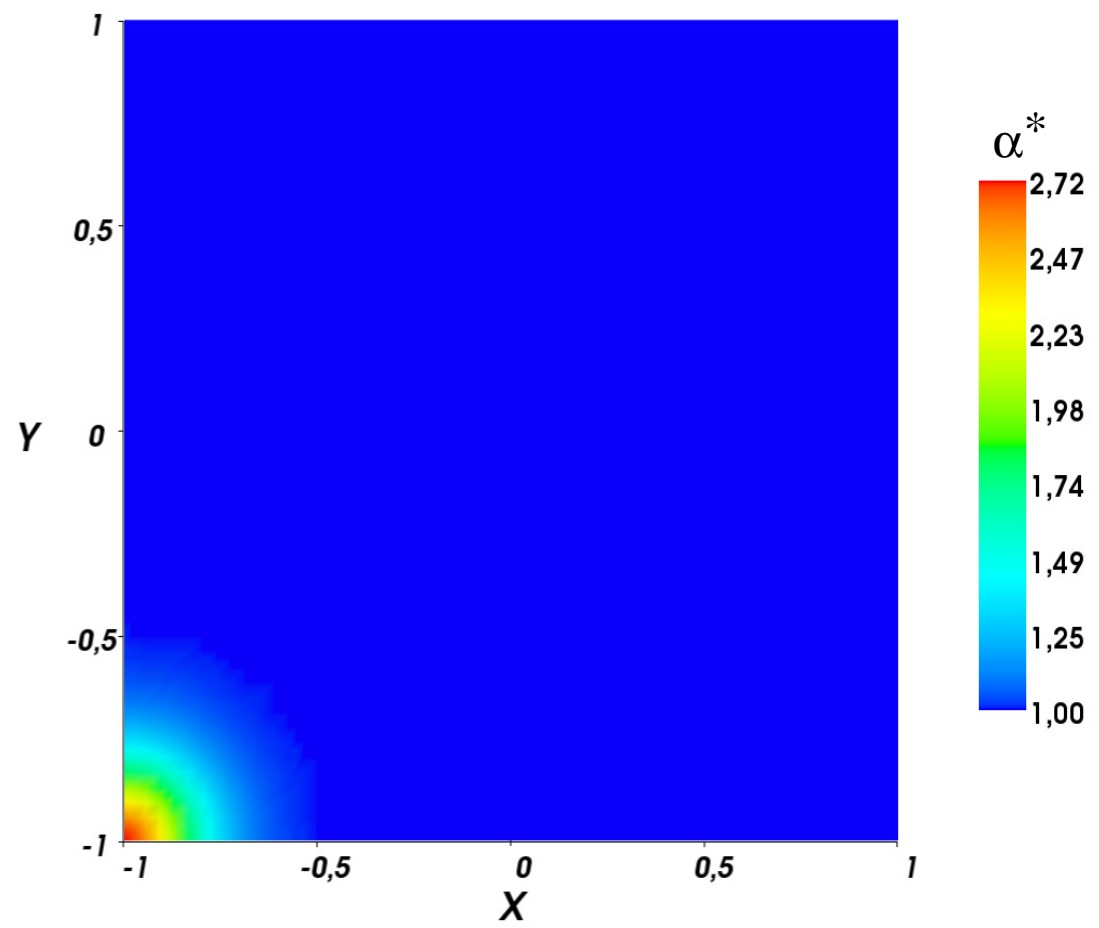

Figure 4. The isovalues of the final function $\alpha^{*}$.

TABLE 1. Comparison of the discretizations with and without simplification.

\begin{tabular}{lcc}
\hline & Without simplification & With simplification \\
\hline Number of iterations & 7 & 9 \\
CPU time(s) & 4.32 & 1.06 \\
\hline
\end{tabular}

We now present in Table 1 the computation time with and without simplification. Even if the number of iterations increases with the simplification, the gain of computational time is undeniable. This proves the interest of our approach.

\section{REFERENCES}

[1] Y. Achdou, C. Bernardi and F. Coquel, A priori and a posteriori analysis of finite volume discretizations of Darcy's equations. Numer. Math. 96 (2003) 17-42.

[2] M. Azaïez, F. Ben Belgacem and C. Bernardi, The mortar spectral element method in domains of operators, Part I: The divergence operator and Darcy's equations. IMA J. Numer. Anal. 26 (2006) 131-154.

[3] M. Azaïez, F. Ben Belgacem, C. Bernardi and N. Chorfi, Spectral discretization of Darcy's equations with pressure dependent porosity. Appl. Math. Comput. 217 (2010) 1838-1856.

[4] M. Azaïez, F. Ben Belgacem, M. Grundmann and H. Khallouf, Staggered grids hybrid-dual spectral element method for secondorder elliptic problems, Application to high-order time splitting methods for Navier-Stokes equations. Comput. Methods Appl. Mech. Engrg. 166 (1998) 183-199.

[5] C. Bernardi, Indicateurs d'erreur en $h-N$ version des éléments spectraux. Modél. Math. et Anal. Numér. 30 (1996) 1-38.

[6] C. Bernardi, A. Blouza, N. Chorfi and N. Kharrat, A penalty algorithm for the spectral element discretization of the Stokes problem. Math. Model. Numer. Anal. 45 (2011) 201-216.

[7] C. Bernardi, T. Chacón Rebollo, F. Hecht and R. Lewandowski, Automatic insertion of a turbulence model in the finite element discretization of the Navier-Stokes equations. Math. Models Methods Appl. Sci. 19 (2009) 1139-1183.

[8] C. Bernardi, F. Coquel and P.-A. Raviart, Automatic coupling and finite element discretization of the Navier-Stokes and heat equations, Internal Report R10001, Labotatoire Jacques-Louis Lions, Paris (2010). 
[9] C. Bernardi, M. Dauge and Y. Maday, Polynomials in Sobolev Spaces and Application to the Mortar Spectral Element Method, in preparation.

[10] C. Bernardi and Y. Maday, Spectral Methods, in the Handbook of Numerical Analysis V, edited by P.G. Ciarlet and J.-L. Lions. North-Holland (1997) 209-485.

[11] C. Bernardi, Y. Maday and F. Rapetti, Discrétisations variationnelles de problèmes aux limites elliptiques, Collection Mathématiques et Applications vol. 45. Springer-Verlag (2004).

[12] M. Braack and A. Ern, A posteriori control of modeling errors and discretization errors. Multiscale Model. Simul. 1 (2003) $221-238$.

[13] H. Brezis and P. Mironescu, Gagliardo-Nirenberg, composition and products in fractional Sobolev spaces, J. Evol. Equ. 1 (2001), 387-404.

[14] F. Brezzi, J. Rappaz and P.-A. Raviart, Finite dimensional approximation of nonlinear problems, Part I: Branches of nonsingular solutions. Numer. Math. 36 (1980) 1-25.

[15] T. Chacón Rebollo, S. Del Pino and D. Yakoubi, An iterative procedure to solve a coupled two-fluids turbulence model. Math. Model. Numer. Anal. 44 (2010) 693-713.

[16] A.L. Chaillou and M. Suri, Computable error estimators for the approximation of nonlinear problems by linearized models. Comput. Methods Appl. Mech. Engrg. 196 (2006) 210-224.

[17] M. Daadaa, Discrétisation spectrale et par éléments spectraux des équations de Darcy, Ph.D. Thesis, Université Pierre et Marie Curie, Paris (2009).

[18] M. Dauge, Neumann and mixed problems on curvilinear polyhedra. Integr. Equ. Oper. Th. 15 (1992) $227-261$.

[19] L. El Alaoui, A. Ern and M. Vohralík, Guaranteed and robust a posteriori error estimates and balancing discretization and linearization errors for monotone nonlinear problems. Comput. Methods Appl. Mech. Engrg. 200 (2011) $2782-2795$.

[20] V. Girault and P.-A. Raviart, Finite Element Methods for Navier-Stokes Equations, Theory and Algorithms. Springer-Verlag (1986).

[21] J.-L. Lions and E. Magenes, Problèmes aux limites non homogènes et applications, Vol. I. Dunod, Paris (1968).

[22] N.G. Meyers, An $L^{p}$-estimate for the gradient of solutions of second order elliptic divergence equations. Ann. Sc. Norm. Sup. Pisa 17 (1963) 189-206.

[23] J. Pousin and J. Rappaz, Consistency, stability, a priori and a posteriori errors for Petrov-Galerkin methods applied to nonlinear problems. Numer. Math. 69 (1994) 213-231.

[24] K.R. Rajagopal, On a hierarchy of approximate models for flows of incompressible fluids through porous solid. Math. Models Methods Appl. Sci. 17 (2007) 215-252.

[25] G. Talenti, Best constant in Sobolev inequality. Ann. Math. Pura ed Appl. Serie IV 110 (1976) 353-372.

[26] R. Verfürth, A Review of A Posteriori Error Estimation and Adaptive Mesh-Refinement Techniques. Wiley ans Teubner (1996). 